Supporting Information for

\title{
Distinct Kinetic Pathways Generate Organogel Networks with Contrasting Fractality and Thixotropic Properties
}

Xiao Huang, ${ }^{1}$ Srinivasa R. Raghavan, ${ }^{2}$ Pierre Terech, ${ }^{3}$ and Richard G. Weiss ${ }^{1}$ *

${ }^{1}$ Department of Chemistry, Georgetown University, 37th and O Streets, NW, Washington, DC 20057-1227, USA, email: weissr@georgetown.edu

${ }^{2}$ Department of Chemical and Biomolecular Engineering, University of Maryland, College Park, MD 20742-2111, USA

${ }^{3}$ CEA-Grenoble, DRFMC/SI3M Laboratoire Physico-Chimie Moleculaire 17, Rue des Martyrs 28054 Grenoble cedex 9, France

\section{$\underline{\text { Experimental section }}$}

Materials. $n$-Octane (Aldrich, 98\%), $n$-dodecane (Acros, 99+\%), $n$-dodecane- $d_{26}$ (MSD Isotopes, 98 atom\% D), acetonitrile (Fisher Scientific, HPLC grade) and ethyl acetate (Fisher Scientific, HPLC grade) were used as received. $5 \alpha$-Cholestan-3 $\beta$-yl $N$-(2-naphthyl) carbamate (CNC) and 3 $\beta$-cholesteryl $N$-(2-naphthyl) carbamate (CeNC), two ALS-type (Aromatic-Linker-Steroid) organic gelators,Error! Bookmark not defined. were synthesized as described in the literature (see below). ${ }^{1}$ CNC was 
recrystallized from ethyl acetate or acetonitrile solutions and $\mathrm{CeNC}$ was recrystallized from 1/4 (v/v) ethyl acetate/hexane.

Syntheses and characterizations of CNC and CeNC. $3 \beta$-Cholesteryl $N$-(2-naphthyl) carbamate (CeNC) was synthesized as described in the literature. ${ }^{2} 0.22 \mathrm{~g}(1.5 \mathrm{mmol})$ 2-aminonaphthalene (Aldrich, 95\%) was dissolved in $7.5 \mathrm{~mL}$ dry benzene with $0.25 \mathrm{~mL}$ dried pyridine in a $50 \mathrm{~mL}$ round-bottom flask. Then, $0.7 \mathrm{~g}(1.6 \mathrm{mmol}) 3 \beta$-cholesteryl chloroformate (Aldrich, 98\%), dissolved in $7.5 \mathrm{~mL}$ dry benzene, was added dropwise with stirring at $0{ }^{\circ} \mathrm{C}$. It was stirred for another $3-4 \mathrm{~h}$, and then extracted with water, saturated aqueous $\mathrm{NaHCO}_{3}$ and water. A yellowish solid was obtained after removal of the liquid using a rotary evaporator under vacuum. The crude product was recrystallized three times from ethyl acetate/hexane $(1 / 4, \mathrm{~V} / \mathrm{V})$ to give $0.32 \mathrm{~g}$ ( $38 \%$ yield) of white crystals, mp $181.2-183.7^{\circ} \mathrm{C}$ (one peak in HPLC with $1 / 9$ ethyl acetate/hexane as eluent). ${ }^{1} \mathrm{H}-\mathrm{NMR}\left(300 \mathrm{MHz}, \mathrm{CDCl}_{3}\right):$ 7.99ppm, 7.78-7.75ppm, 7.47-7.26 ppm (m, 7H, aromatic), 6.70ppm (s, 1H, NH), $5.43(\mathrm{~s}, 1 \mathrm{H}, \mathrm{C}=\mathrm{C}-\mathrm{H}), 4.67-4.61 \mathrm{ppm}(\mathrm{m}, 1 \mathrm{H}, \mathrm{CH}), 2.46-0.60 \mathrm{ppm}$ (m, 44H, cholesteryl). IR (Attenuated total reflectance): 3426 (N-H), 3060 (aromatic, C-H $), 1731(\mathrm{C}=\mathrm{O}, \mathrm{s}), 1644(\mathrm{C}=\mathrm{C}) \mathrm{cm}^{-1}$. Elemental analysis: calcd for $\mathrm{C}_{38} \mathrm{H}_{53} \mathrm{NO}_{2}, \mathrm{C}$ $82.11 \%$, H 9.61\%, N 2.52\%; found, C 81.83\%, H 9.90\%, N 2.77\%. $5 \alpha$-Cholestan-3 $\beta$-yl $N$-(2-naphthyl) carbamate (CNC) was synthesized similarly (using $5 \alpha$-cholestan-3 $\beta$-yl chloroformate) to yield material of mp $184.1-184.9^{\circ} \mathrm{C}$ (lit mp 178 $180{ }^{\circ} \mathrm{C}^{2}$; one peak in HPLC with 1/9 ethyl acetate/hexane as eluent). Elemental Analysis: 
calcd for $\mathrm{C}_{38} \mathrm{H}_{55} \mathrm{NO}_{2}, \mathrm{C} 81.82 \%, \mathrm{H} 9.94 \%, \mathrm{~N} 2.51 \%$; found, C 81.83\%, H 10.38\%, N

2.53\%. ${ }^{1} \mathrm{H}-\mathrm{NMR}: 8.00,7.80-7.72,7.46-7.28$ (m, 7H, aromatic), $6.72(\mathrm{~s}, 1 \mathrm{H}, \mathrm{NH})$,

4.80-4.65 (m, 1H, CH), 2.20-0.60 ppm (m, 46H, cholestanyl).

Supporting Table 1. Sources and Purities of Liquids for Gelation Tests

\begin{tabular}{lll}
\hline Solvent & Purity (from supplier) & Supplier \\
\hline methanol & $99+\%$ & Aldrich \\
ethanol & 200 proof & Warner-Graham \\
1 -propanol & certified & Fisher Scientific \\
1 -butanol & $99.5 \%$, spectrophotometric & Aldrich \\
1 -pentanol & certified & Fisher Scientific \\
1 -octanol & $99+\%$ & Aldrich \\
$n$-octane & $98 \%$ & Aldrich \\
$n$-decane & $99+\%$ & Acros \\
$n$-dodecane & $99+\%$ & Acros \\
cyclohexane & HPLC & Fisher Scientific \\
benzene & certified & Fisher Scientific \\
acetonitrile & HPLC & Fisher Scientific \\
ethyl acetate & HPLC & Fisher Scientific \\
chloroform & FPLC & Fisher Scientific \\
\hline
\end{tabular}

Instrumentation and sample preparations. Samples for gelation studies were prepared by flame-sealing $5 \mathrm{~mm}$ (i.d.) glass tubes that contained known amounts of a liquid and gelator. The tubes were heated in a water or oil bath until the gelator dissolved completely. Thereafter, the hot solutions/sols were cooled rapidly to different 
temperatures and incubated there until gelation occurred. Qualitatively, gelation was considered successful if no sample flow was observed upon inverting the container at room temperature (i.e., the 'inverse flow' method ${ }^{3}$ ) after a third heating-and-cooling cycle. Sources and purity of liquids employed in gelation test are listed in Supporting Figure 1.

Optical micrographs (OMs) and melting points were recorded on a Leitz 585

SM-LUX-POL microscope equipped with crossed polars, a Photometrics CCD camera interfaced to a computer, a Leitz 350 heating stage, and an Omega HH503 microprocessor thermometer connected to a J-K-T thermocouple. The samples for optical micrographs were aliquots of gels described above that had been transferred as sols to 0.4 mm thickness flatted capillary tubes (VitroCom) and flame-sealed therein. They were then heated to their solution/sol phase in a boiling water bath and incubated at different temperatures until gelation occurred before the OMs were recorded.

X-ray diffraction (XRD) of samples was performed on a Rigaku R-AXIS image-plate system with $\mathrm{Cu} \mathrm{Kx}$ X-rays $(\AA=1.54 \AA)$ generated by a Rigaku generator operating at 46 $\mathrm{kV}$ and $46 \mathrm{~mA}$ with the collimator at $0.3 \mathrm{~mm}$. Data processing and analyses were performed using Materials Data JADE (version 5.0.35) XRD pattern processing software. Samples were sealed in either $1.0 \mathrm{~mm}$ (gels, exposures for $10 \mathrm{~h}$ ) or $0.5 \mathrm{~mm}$ (neat powder or xerogel, exposure for 15 min) glass capillaries (W. Müller, Schönwalde, Germany). CNC xerogels were prepared by drying fresh $\mathbf{C N C} / n$-octane gels (defined as samples within $5 \mathrm{~h}$ of initial cooling) under a dynamic reduced pressure of 440 Torr for several 
days. This process yielded samples that were not thoroughly dried, as indicated by a broad peak around $20^{\circ}$ in $2 \theta$ in XRD patterns and thermal gravimetric analyses (TGA).

Differential scanning calorimetry (DSC) was performed on a TA 2910 differential scanning calorimeter interfaced to a TA Thermal Analyst 3100 controller. All DSC temperatures are reported at maximum heat flow. Heating rates were $5{ }^{\circ} \mathrm{C} / \mathrm{min}$; cooling rates were variable and depended on the difference between the cellblock and ambient temperatures.

Kinetics of gelation by CD spectroscopy. CNC, dissolved in hot $n$-octane, was transferred to a $1.0 \mathrm{~mm}$ thick water-jacketed $\mathrm{CD}$ cell that was kept at $90{ }^{\circ} \mathrm{C} . \mathrm{CD}$ spectra were recorded on a Jasco-700 CD spectrometer immediately after liquid, at a predetermined temperature, was flowed from a thermostatting circulating bath through the jacket of the cell. The approximate period required for temperature equilibration after sample transfer was less than 30 seconds since a very small amount of sample was used. The shortest period to complete gelation was 10-15 min. The scan rate and other instrumental parameters were set to optimize the signal-to-noise ratio. Contributions from linear dichroism are negligible since only slight changes in CD intensity were detected when samples were rotated by $90^{\circ}$. Experiments with $\mathbf{C e N C} /$ ethyl acetate samples were performed in the same way except that the cell was kept at $70{ }^{\circ} \mathrm{C}$ before the hot sols were transferred into the cells.

Kinetics of gelation by fluorescence spectroscopy. $\mathbf{C N C}$ and $n$-octane were heated until all solids dissolved in a sealed $3 \mathrm{~mm}$ pathlength flattened quartz tube (VitroCom). Then, 
the tube was placed into a thermostatted cell holder of the fluorimeter (with a VWR-1140 circulating thermostatting bath) and the emission intensity at $375 \mathrm{~nm}$ (excitation at 318 $\mathrm{nm})$ was recorded from the front-face as a function of time at a rate of 1 datum per second on Spex Fluorolog III spectrometer. In the data treatment, sets of 10 or 100 consecutive points were averaged and fitted to the kinetic models. The same procedures were employed for preparation of $\mathbf{C e N C / e t h y l ~ a c e t a t e ~ s a m p l e s . ~ T h e ~ e x c i t a t i o n ~ a n d ~}$ emission wavelengths were 333 and $358 \mathrm{~nm}$, respectively, and data were recorded on a PTI Qm-Double EX spectrometer equipped with A TLC 50 Fluorescence cuvette holder, a Peltier thermoelectric temperature controller, and Eheim 2028 water circulator.

Kinetics of gelation by small angle neutron scattering (SANS). Data were collected on the $30 \mathrm{~m}$ SANS beam line at the National Center for Neutron Research of the National Institute of Standards and Technology, Gaithersburg, MD (5 m scattering, $\lambda=8.09 \AA$ ) using $2 \mathrm{~mm}$ pathlength cylindrical quartz cells placed in a thermostatted $\left( \pm 0.1^{\circ} \mathrm{C}\right)$ cell holder. Hot samples of $\mathbf{C N C}$ with $n$-dodecane- $d_{26}$ as the liquid were transferred to the thermostatted cells as described above. The delay time between the transfer of sample and initial measurements was less than $10 \mathrm{~s}$. In the kinetic runs, intensity data collected in a solid angle defined by a $Q$ range of $0.008-0.1 \AA^{-1}$ were summed during $10 \mathrm{~s}$ increments with $35 \mathrm{~s}$ delays between collection periods. The sums were plotted then as a function of time to obtain the kinetic parameters as explained in the text. The raw data for the kinetic runs are included in Supporting Table 7.

Kinetics of gelation and thixotropic studies by rheological measurements. Rheology data 
were recorded on a Rheometric Scientific RDA-III strain-controlled rheometer using Peltier-controlled parallel plates ( $25 \mathrm{~mm}$ diameter). The sample temperature was controlled to $\pm 0.1{ }^{\circ} \mathrm{C}$. Experiments were conducted under oscillatory shear, and the storage modulus $\left(G^{\prime}\right)$, the loss modulus $\left(G^{\prime \prime}\right)$, the ratio between $G^{\prime \prime}$ and $G^{\prime}(\tan \delta)$, and the complex viscosity $\left(\eta^{*}\right)$ were measured as functions of frequency, $\omega$. The gap between the plates $(0.3-0.5 \mathrm{~mm})$ did not influence the rheological data, indicating that wall slip was not a factor.

All rheological experiments were performed with $n$-dodecane, rather than $n$-octane, as the liquid to minimize evaporation from samples at super-ambient temperatures, and at a strain of $1 \%$ and a frequency of $10 \mathrm{rad} / \mathrm{s}$, which are within the approximate linear viscoelastic (LVE) regime ${ }^{4}$ (Supporting Figure 1); at $40^{\circ} \mathrm{C}$, slightly below the sol-gel transition temperature, the frequency sweeps indicate that the sample behaves like a soft viscoelastic solid. To reduce evaporation of the solvent further, a plastic cover with a liner soaked with $n$-dodecane was placed over the apparatus when measurements were made at 35 or $40{ }^{\circ} \mathrm{C}$. Hot solutions of $\mathbf{C N C}$ in $n$-dodecane were transferred onto the lower plate that was at $40{ }^{\circ} \mathrm{C}$ when the desired temperature was higher than $20^{\circ} \mathrm{C}$, or at $20{ }^{\circ} \mathrm{C}$ higher than the desired temperature when the desired temperature was below 20 ${ }^{\circ} \mathrm{C}$. The plate was cooled quickly (ca. $30 \mathrm{~s}$ ) to the desired temperature after the plate gap was adjusted to the desired separation. Recording of kinetic data commenced immediately thereafter. 
During rheological measurements to determine thixotropic properties, samples were left undisturbed for $30 \mathrm{~min}$ to $4 \mathrm{~h}$ (based on the nature of the kinetic study) to complete gelation after the temperature and the gap reached the desired values. The sample was then sheared at a constant shear rate of $2 \mathrm{~s}^{-1}$ for $600 \mathrm{~s}$ and immediately afterwards, the recovery of $G$ ' was monitored. A frequency-dependent sweep was also performed both before shearing and after recovery between $0.01-100 \mathrm{rad} / \mathrm{s}$ at $1 \%$ strain to ascertain whether a 'true' gel was present. ${ }^{5}$ After one or two shear-recovery cycles at $25^{\circ} \mathrm{C}$ were completed, the plates were separated, a small portion of sample was transferred onto a cover glass with minimal mechanical disturbance using a micro-spatula, and optical micrographs were recorded. 

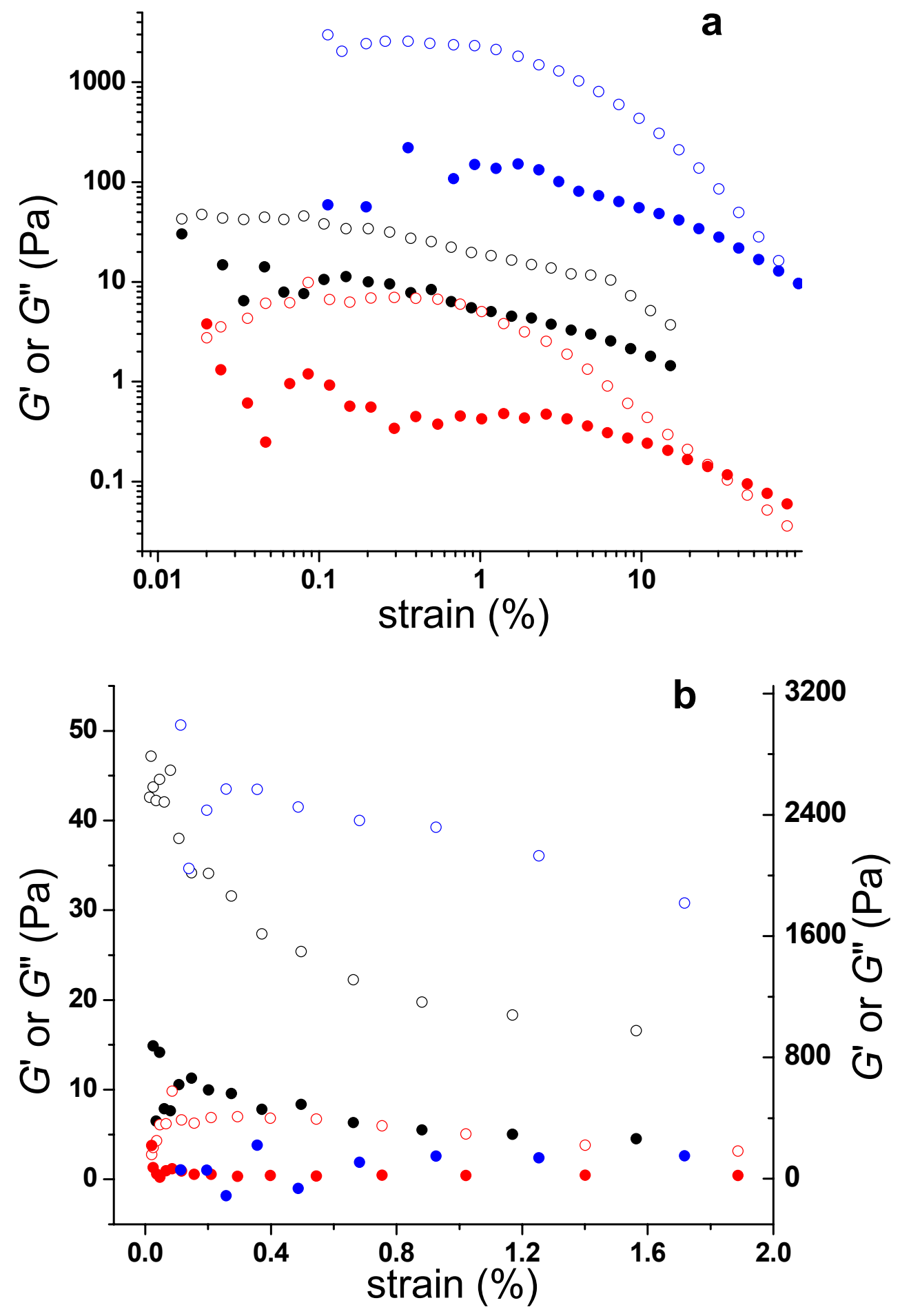


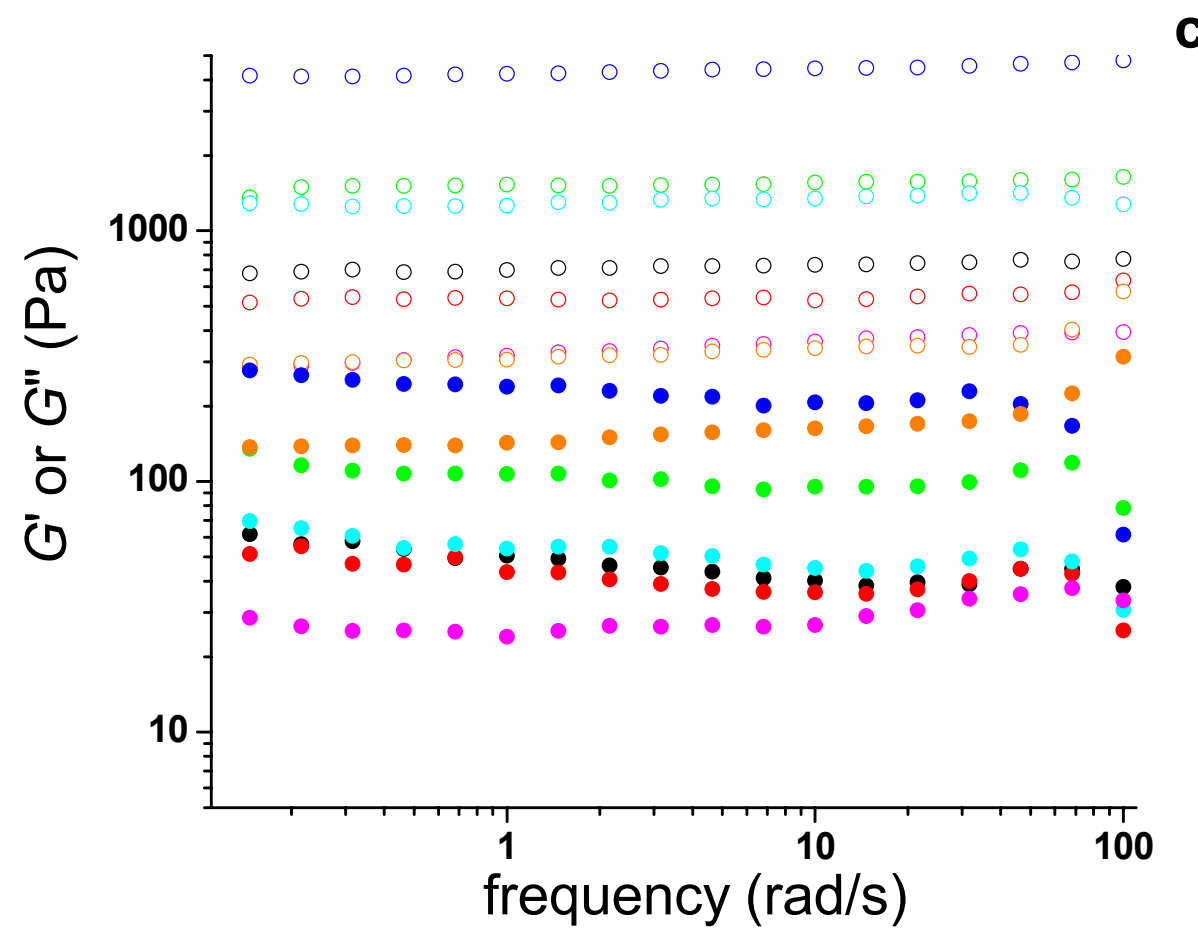

Supporting Figure 1. Log-log (a) and linear (b) strain and frequency (c) sweeps for 1.0 $\mathrm{wt} \% \mathrm{CNC} / n$-dodecane gels performed at the temperatures at which the gels were formed (except in the strain sweeps of the gel formed at $40{ }^{\circ} \mathrm{C}$ and measured at $25^{\circ} \mathrm{C}(\bullet, \circ)$ ). Unfilled symbols are $G^{\prime}$ data; filled symbols are $G$ '”. (a,b) Gels formed by incubation of sols at $0\left(\bullet, \circ\right.$, left Y-axis) and $40^{\circ} \mathrm{C}(\bullet, \circ$, left Y-axis; $\bullet$,, , right Y-axis). (c) Gels formed by incubation of sols at $0(\bullet, \circ), 5(\bullet, \circ), 10(\bullet, \circ), 15(\bullet, \circ), 25(\bullet, \circ), 30(\bullet, \circ)$ and $40{ }^{\circ} \mathrm{C}(\bullet, \circ)$.

Supporting Table 2. Gelation studies of $\mathrm{CeNC}^{\mathrm{a}}$

\begin{tabular}{|c|c|c|c|c|}
\hline$w t \%$ & Liquid & $\begin{array}{l}\text { Fast cooling of } \\
\text { sol (ice-water } \\
\text { bath) }\end{array}$ & $\begin{array}{l}\text { Moderate cooling of } \\
\text { sol (in air) }\end{array}$ & $\begin{array}{l}\text { Slow cooling of sol } \\
\text { (left in hot water bath } \\
\text { that returned slowly to } \\
\text { RT) }\end{array}$ \\
\hline 3 & $n$-octane & $\mathrm{ppt}$ & $\mathrm{ppt}$ & Ppt \\
\hline 3 & $n$-decane & PG & PG & PG \\
\hline 3 & n-dodecane & PG & PG & PG \\
\hline 3 & cyclohexane & - & ppt & Ppt \\
\hline
\end{tabular}




\begin{tabular}{|c|c|c|c|c|}
\hline 3 & $\mathrm{CHCl}_{3}$ & Sol & Sol & Sol \\
\hline 3 & benzene & - & Sol & Sol \\
\hline 3 & toluene & $\mathrm{G}(<\mathrm{RT})$ & Sol & Sol \\
\hline 3 & $\mathrm{CH}_{3} \mathrm{CN}$ & $\mathrm{ppt}$ & $\mathrm{ppt}$ & Ppt \\
\hline 3 & ethyl acetate & G (39.7-46.1) & $\mathrm{G}(40.2-42.6)$ & $\mathrm{G}(43.5-43.7)$ \\
\hline 2 & ethyl acetate & $\mathrm{G}(40.2-44.7)$ & $\mathrm{G}(38.0-40.2)$ & G (39.9-40.4) \\
\hline 1 & ethyl acetate & $\mathrm{ppt}$ & ppt & Ppt \\
\hline 0.5 & ethyl acetate & Sol & Sol & Sol \\
\hline 0.2 & methanol & ppt & ppt & $\begin{array}{l}\text { Microcrystal } \\
\text { dispersion }^{\mathrm{b}}\end{array}$ \\
\hline 0.5 & methanol & ppt & ppt & $\begin{array}{l}\text { Microcrystal } \\
\text { dispersion }{ }^{b}\end{array}$ \\
\hline 1.0 & methanol & $\mathrm{ppt}$ & ppt & $\begin{array}{l}\text { Microcrystal } \\
\text { dispersion }^{b}\end{array}$ \\
\hline 1.0 & 1-propanol & ppt & $\mathrm{G}(35.1-42.5)$ & $\mathrm{G}(44.5-45.0)$ \\
\hline 1.0 & 1-butanol & $\mathrm{ppt}$ & $\mathrm{G}(30.0-30.5)$ & $\mathrm{G}(30.3-42.9)$ \\
\hline 1.0 & 1-pentanol & PG & $\mathrm{G}(38.0-38.8)$ & G (38.8-43.0) \\
\hline 1.0 & glycerol & $\mathrm{ppt}$ & ppt & $\mathrm{G}(73.5-76.7)$ \\
\hline 1.0 & $\begin{array}{l}\text { benzyl } \\
\text { alcohol }\end{array}$ & $\mathrm{G}(\sim \mathrm{RT})$ & $\mathrm{G}(49.3-55.2)$ & $\mathrm{G}(32.0-32.5)$ \\
\hline 3.0 & ethanol & $\mathrm{G}(86.3-89.1)$ & $\mathrm{G}(88.0-90.7)$ & $\mathrm{G}(87.2-87.9)$ \\
\hline 1.0 & ethanol & ppt & ppt & $\begin{array}{l}\text { Microcrystal } \\
\text { dispersion }^{\mathrm{b}}\end{array}$ \\
\hline 0.5 & ethanol & $\mathrm{ppt}$ & $\begin{array}{l}\text { Microcrystal } \\
\text { dispersion }{ }^{\mathrm{b}}\end{array}$ & $\begin{array}{l}\text { Microcrystal } \\
\text { dispersion }^{b}\end{array}$ \\
\hline 3.0 & 1-octanol & $\mathrm{ppt}$ & $\mathrm{G}(59.1-71.5)$ & $\mathrm{G}(59.9-62.5)$ \\
\hline 2.0 & 1-octanol & $\mathrm{ppt}$ & $\mathrm{G}(47.8-56.3)$ & $\mathrm{PG}$ \\
\hline 1.0 & 1-octanol & some ppt & some ppt & some ppt \\
\hline
\end{tabular}

${ }^{a}$ G: gel; PG: partial gel; ppt: precipitate; Sol: solution; The values in parentheses are gel-to-sol transition temperatures, $\mathrm{T}_{\mathrm{g}}$, determined by the inverse flow method: sealed tubes with gel samples were inverted and placed next to a thermometer in a water bath at 
room temperature and the water bath was stirred and heated. The temperature range for the gel-to-sol transition was taken to be from the moment of initial sample flow to when it fell completely to the bottom of the tube. ${ }^{b}$ phase-separated sample of microcrystallites that, when inverted, does not fall.

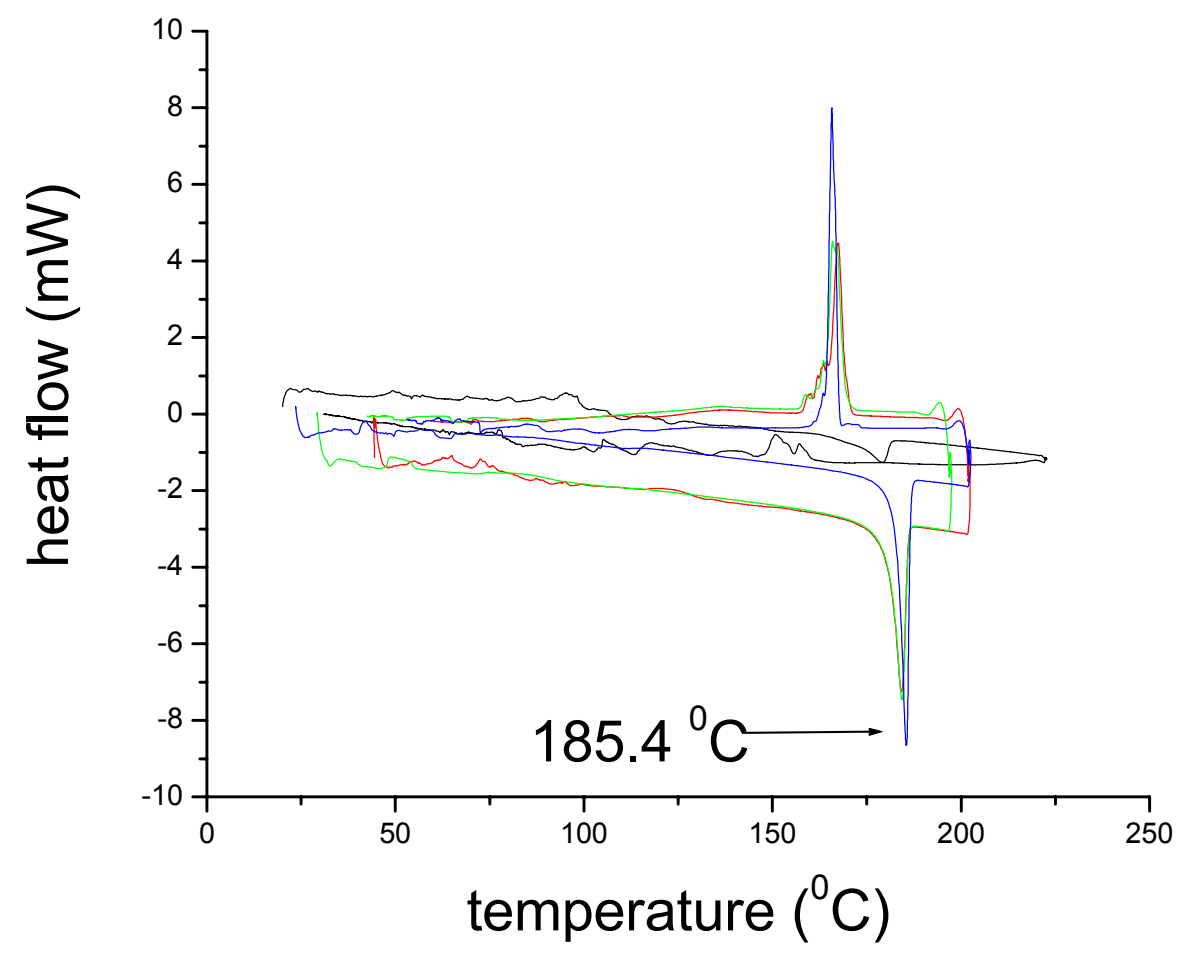

Supporting Figure 2. DSC of CNC fiber-like crystal obtained from ethyl acetate (-) and of $\mathbf{C N C}$ spherulitic solid obtained by drying a MeCN solution (first run, —; second run, - ; third run, - -). 

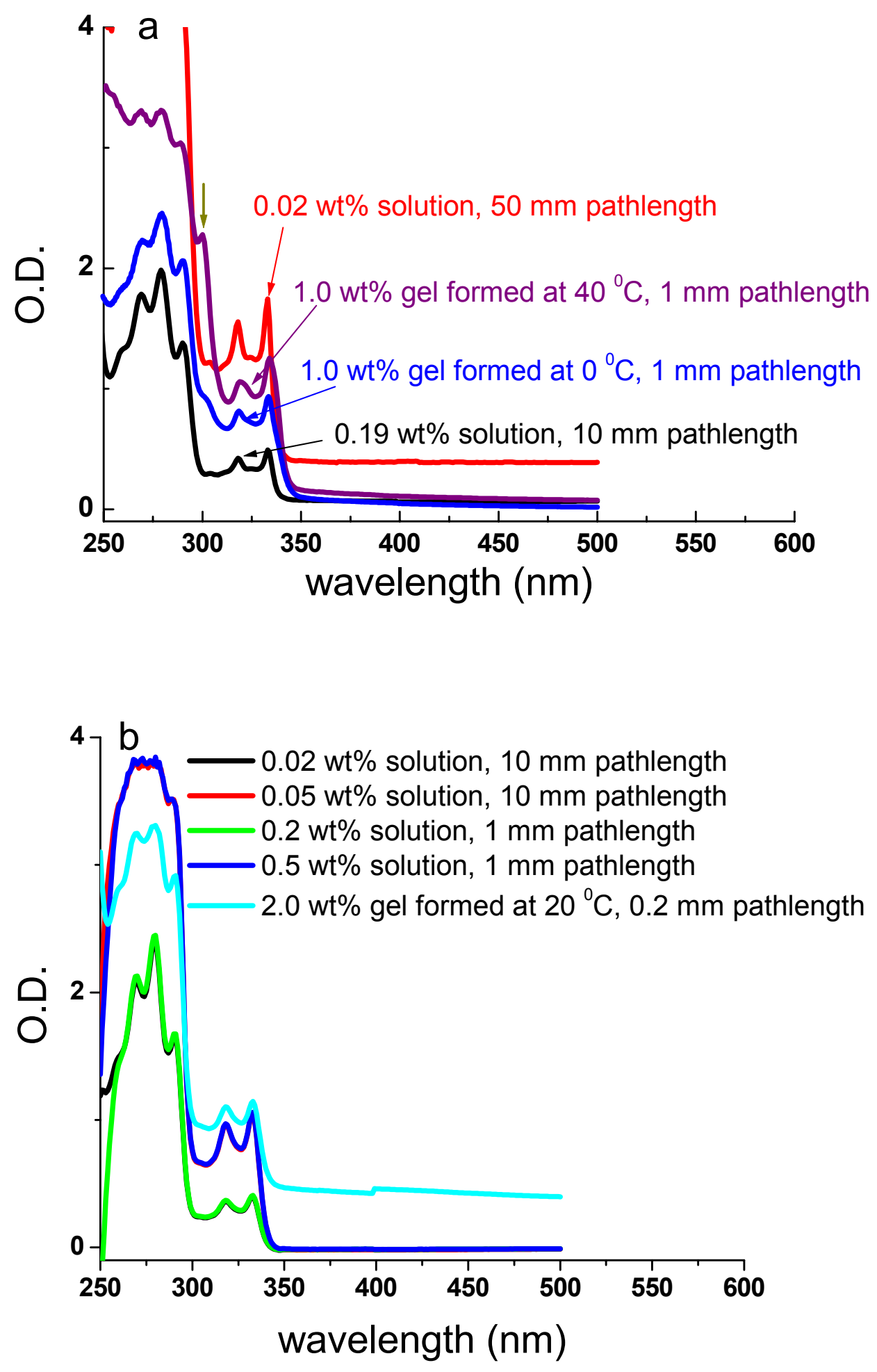

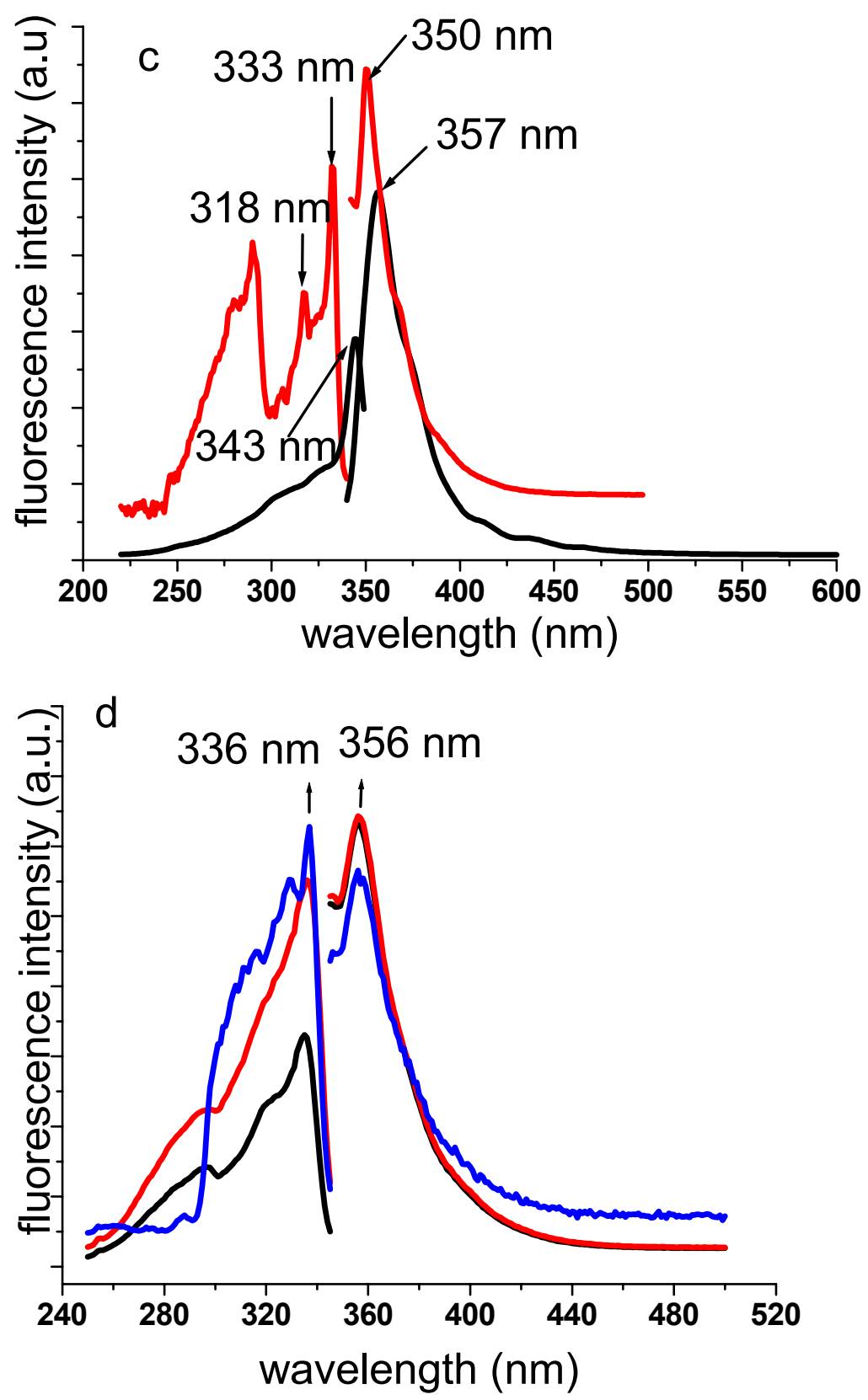

Supporting Figure 3. Absorption spectra at room temperature of a) CNC in $n$-octane ${ }^{6}$ and b) CeNC in ethyl acetate. Data for O.D. values above 3 are inaccurate. c) Emission (right) and excitation (left) spectra of an $0.02 \mathrm{wt} \% \mathbf{C N C} / n$-octane $\mathrm{N}_{2}$-saturated solution (-, $\lambda_{\text {ex }} 318 \mathrm{~nm}$ and $\lambda_{\text {em }} 350 \mathrm{~nm}$; right-angle geometry) and a $1.0 \mathrm{wt} \% \mathbf{C N C} / n$-octane gel in air formed by incubation of the corresponding sol at $25^{\circ} \mathrm{C}\left(-, \lambda_{\text {ex }} 333 \mathrm{~nm}\right.$ and $\lambda_{\text {ex }} 357$ 
$\mathrm{nm}$; front-face geometry); d) Emission (right) and excitation (left) spectra of $0.01 \mathrm{wt} \%$ (-) and $0.5 \mathrm{wt} \%$ (-) CeNC/ethyl acetate solutions ( $\lambda_{\mathrm{ex}} 333 \mathrm{~nm}$ and $\lambda_{\mathrm{em}} 356 \mathrm{~nm}$; right-angle geometry) and a $2.0 \mathrm{wt} \% \mathrm{CeNC} /$ ethyl acetate gel in air formed by incubation of the corresponding sol at $0{ }^{\circ} \mathrm{C}\left(-, \lambda_{\mathrm{ex}} 333 \mathrm{~nm}\right.$ and $\lambda_{\mathrm{ex}} 356 \mathrm{~nm}$; front-face geometry). The wavelengths in the figures are the intensity maxima.

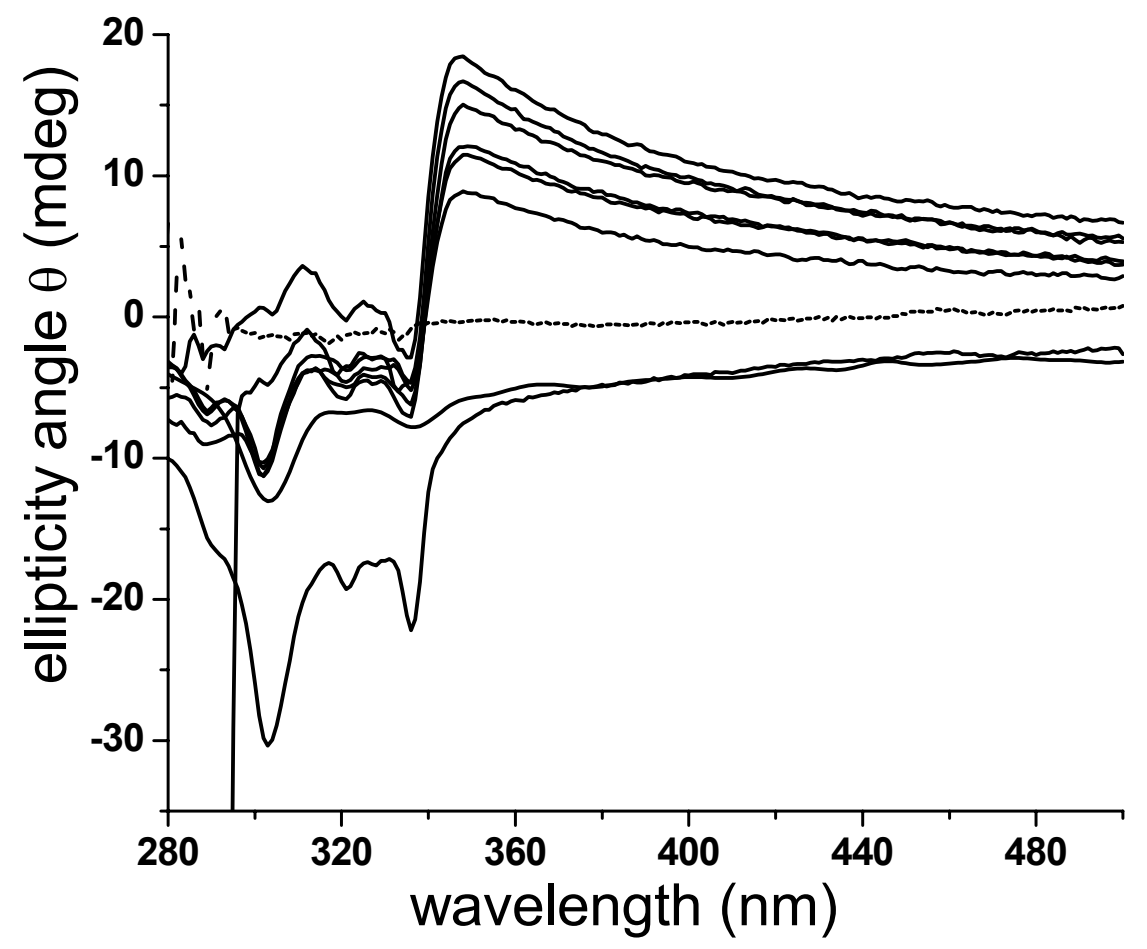

Supporting Figure 4. CD spectra of a $0.02 \mathrm{wt} \% \mathrm{CNC} / n$-octane sol at room temperature (50 mm pathlength cell; dashed line) and $1.0 \mathrm{wt} \% \mathbf{C N C} / n$-octane gels ( $1 \mathrm{~mm}$ pathlength cell) formed by cooling the sol rapidly from well above $\mathrm{T}_{\mathrm{g}}$ and incubating the sample at $5.4,8.7,12.1,20.2,25.0,30.0,35.0$ and $40.0^{\circ} \mathrm{C}$ (solid lines, ellipticities from top to bottom at $346 \mathrm{~nm})^{6}$ 

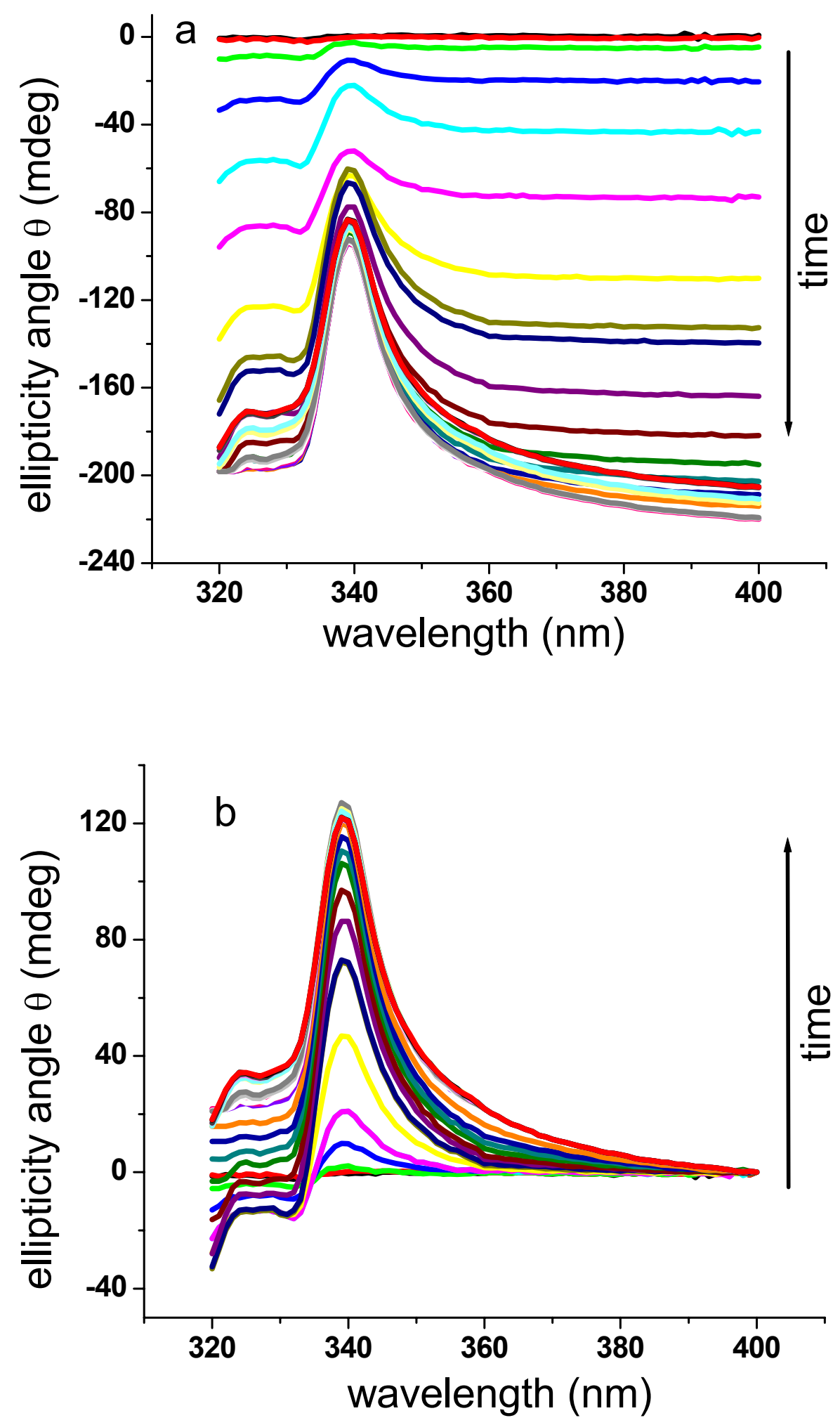

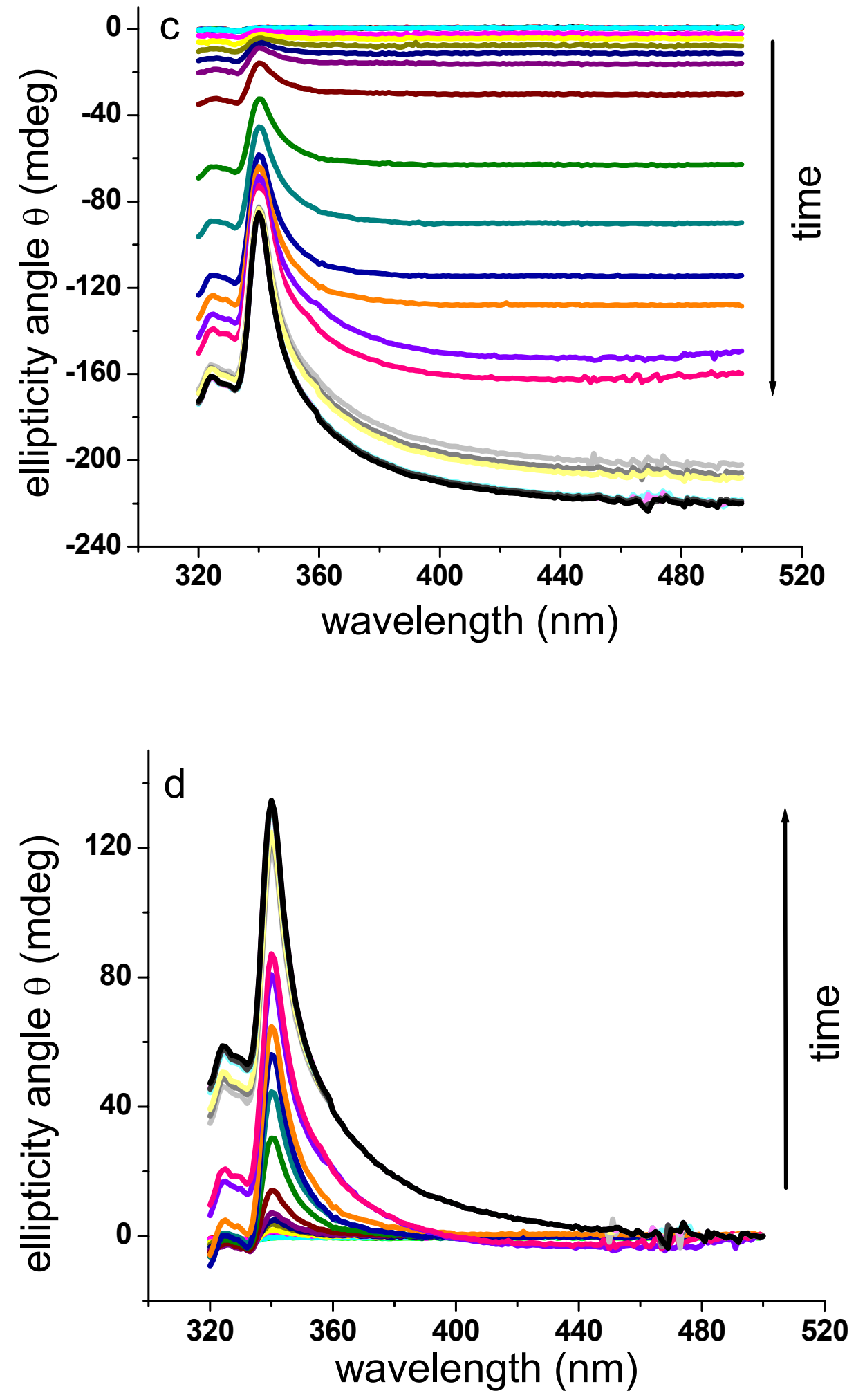

Supporting Figure 5. CD spectra of $2.0 \mathrm{wt} \% \mathrm{CeNC} / \mathrm{ethyl}$ acetate hot sols cooled rapidly 
to and incubated at 0 in $0.2 \mathrm{~mm}$ thick cell (a, b; intervals between the beginning and end of data collection for each sequential spectrum (an accumulation of several scans, each requiring 15 s) were $90,90,90,90,90,90,90,62,90,90,90,90,95,90,90,90,90,241$, $150,995,150,1954,150$, and $1558 \mathrm{~s}$; the total time was $6742.5 \mathrm{~s})$ or at $20^{\circ} \mathrm{C}(\mathrm{c}, \mathrm{d}$; intervals between the beginning and end of data collection for each sequential spectrum (an accumulation of several scans, each requiring $55 \mathrm{~s}$ ) were 825, 3050.5, 550, 550, 1962, $330,330,330,330,503,1174,1003,1003,330,330,330,330,1187,550,550,2424,550$, 550 , and $986 \mathrm{~s}$; the total time is $20342.5 \mathrm{~s}$ ) to follow gel formation. In (b) and (d), the spectra have been offset to normalize ellipticities to 0 degrees at 400 (b) or $500 \mathrm{~nm}$ (d).

Supporting Table 3. Summary of fractal dimensions of SAFINs from $1.0 \mathrm{wt} \%$ $\mathrm{CNC} / n$-alkane gels formed by incubation of sols at different temperatures using data from different techniques.

\begin{tabular}{|l|l|l|}
\hline \multirow{3}{*}{ Temp $\left( \pm 0.1^{\circ} \mathrm{C}\right)$} & $D_{f}$ & $\mathrm{R}^{2}$ \\
\cline { 2 - 3 } & (from fluorescence) & \\
\hline 1.1 & 1.74 & 0.993 \\
\hline 3.8 & 1.72 & 0.993 \\
\hline 9.3 & 1.75 & 0.992 \\
\hline 13.9 & 1.70 & 0.994 \\
\hline 22.5 & 1.79 & 0.991 \\
\hline 23.8 & 1.80 & 0.992 \\
\hline 32.0 & 1.42 & 0.983 \\
\hline 32.2 & 1.38 & 0.992 \\
\hline 39.2 & 1.38 & 0.988 \\
\hline & & \\
\hline 40.0 & $($ from SANS $)$ & \\
\hline & 1.38 & 0.997 \\
\hline 5.0 & & \\
\hline 8.0 & (from CD) & 0.989 \\
\hline 12.0 & 1.72 & 0.985 \\
\hline 16.0 & 1.73 & 0.989 \\
\hline 20.0 & 1.68 & 0.991 \\
\hline 25.0 & 1.71 & 0.976 \\
\hline 30.0 & 1.75 & 0.996 \\
\hline 40.0 & 1.64 & 0.993 \\
\hline & $1.37(304 \mathrm{~nm})$ & 0.982 \\
\hline & $1.42(336 \mathrm{~nm})$ & 0.992 \\
\hline & & \\
\hline & & \\
\hline & & \\
\hline
\end{tabular}




\begin{tabular}{|l|l|l|} 
& (from rheology) & \\
\hline 0.0 & 1.78 & 0.998 \\
\hline 5.0 & 1.70 & 0.996 \\
\hline 10.0 & 1.75 & 0.991 \\
\hline 15.0 & 1.73 & 0.991 \\
\hline 30.0 & 1.36 & 0.991 \\
\hline 35.0 & 1.38 & 0.998 \\
\hline 40.0 & 1.38 & 0.998 \\
\hline
\end{tabular}

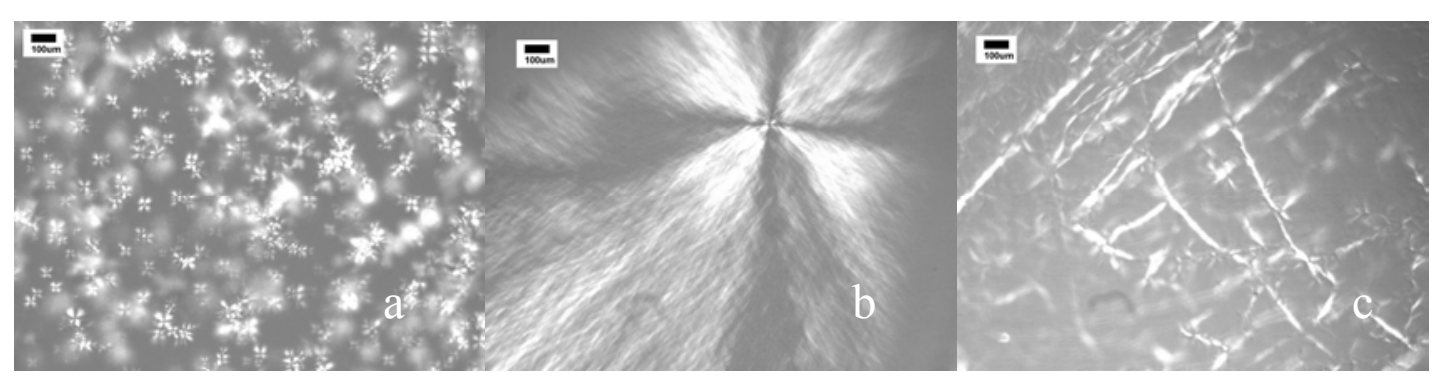

Supporting Figure 6. Optical micrographs of $1.0 \mathrm{wt} \% \mathrm{CNC} / n$-octane gels formed at 0 , 25.1 and $37.4{ }^{\circ} \mathrm{C} .{ }^{6}$ Scale bars $=100 \mu \mathrm{m}$.

\begin{tabular}{|c|c|c|}
\hline \multicolumn{3}{|c|}{$\begin{array}{l}\text { Supporting Table 4. Summary of fractal dimensions of SAFINs in gels from } \\
\text { incubation of sols at } 32.2^{\circ} \mathrm{C} \text { with different concentrations of } \mathrm{CNC} / n \text {-octane using } \\
\text { data from fluorescence. }\end{array}$} \\
\hline$[\mathrm{CNC}](\mathrm{wt} \%)$ & $D_{f}$ & $\mathrm{R}^{2}$ \\
\hline 3.0 & 1.76 & 0.981 \\
\hline 2.5 & 1.73 & 0.992 \\
\hline 1.94 & 1.72 & 0.991 \\
\hline 1.46 & 1.69 & 0.991 \\
\hline 1.0 & 1.38 & 0.992 \\
\hline 0.89 & 1.32 & 0.993 \\
\hline
\end{tabular}

Supporting Table 5. Summary of fractal dimensions and Avrami parameters for $2.0 \mathrm{wt} \% \mathrm{CeNC} / \mathrm{ethyl}$ acetate gels formed by incubation of sols at various temperatures from data using different techniques.

\begin{tabular}{|l|l|l|l|l|l|}
\hline Temperature $\left( \pm 0.1^{\circ} \mathrm{C}\right)$ & $n$ & $K\left(\mathrm{~s}^{-1}\right)$ & $\mathrm{R}^{2}$ & $D_{f}$ & $\mathrm{R}^{2}$ \\
\hline (from fluorescence) & & & & & \\
\hline 21.9 & 2.05 & -15.3 & 0.995 & 1.15 & 0.994 \\
\hline
\end{tabular}




\begin{tabular}{|l|l|l|l|l|l|}
\hline 20.5 & 2.07 & -15.0 & 0.996 & 1.14 & 0.995 \\
\hline 20.4 & 1.92 & -18.6 & 0.999 & 1.14 & 0.999 \\
\hline 16.1 & 2.06 & -13.0 & 0.994 & 1.14 & 0.988 \\
\hline 12.0 & 1.99 & -13.7 & 0.994 & 1.30 & 0.988 \\
\hline 11.4 & 2.12 & -15.3 & 0.994 & 1.15 & 0.997 \\
\hline 8.2 & 2.00 & -11.4 & 0.995 & 1.29 & 0.986 \\
\hline 7.0 & 2.08 & -15.1 & 0.997 & 1.12 & 0.995 \\
\hline 3.4 & 2.07 & -12.2 & 0.995 & 1.16 & 0.997 \\
\hline 2.5 & 1.73 & -12.2 & 0.993 & 1.24 & 0.998 \\
\hline 0.3 & 1.07 & -6.45 & 0.996 & 1.30 & 0.996 \\
\hline-2.6 & 1.12 & -5.55 & 0.995 & 1.29 & 0.988 \\
\hline-4.1 & 1.17 & -6.87 & 0.991 & 1.29 & 0.986 \\
\hline-7.3 & 1.05 & -8.00 & 0.993 & 1.30 & 0.999 \\
\hline-8.4 & 1.06 & -6.33 & 0.992 & 1.27 & 0.992 \\
\hline$($ from CD) & & & & & \\
\hline 20.2 & 2.09 & -18.2 & 0.990 & 1.11 & 0.992 \\
\hline 0.6 & 1.89 & -12.2 & 0.993 & 1.23 & 0.986 \\
\hline
\end{tabular}



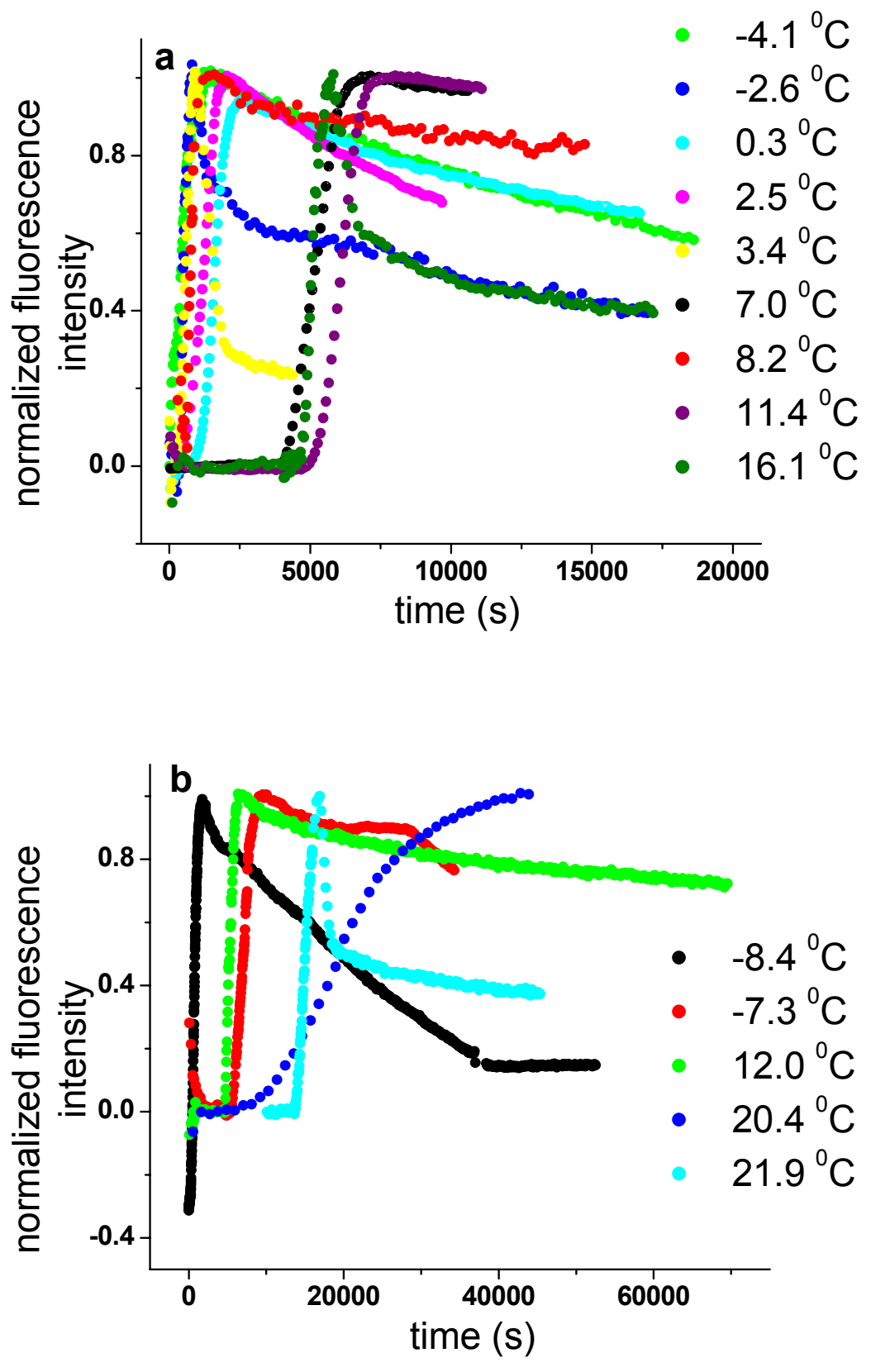


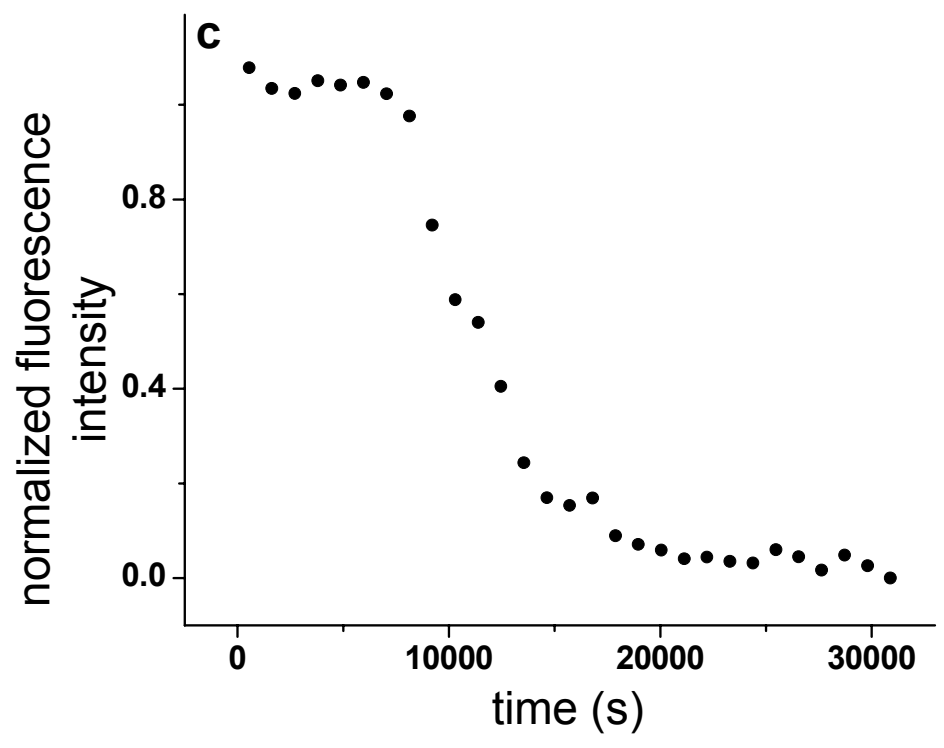

Supporting Figure 7. Normalized fluorescence intensity $\left(\lambda_{\mathrm{em}} 358 \mathrm{~nm} ; \lambda_{\mathrm{ex}} 333 \mathrm{~nm}\right)$ changes versus time for sols cooled rapidly to and incubated at the temperatures indicated in (a, b) $2.0 \mathrm{wt} \% \mathrm{CeNC} /$ ethyl acetate or at $24.9^{\circ} \mathrm{C}$ in (c) $2.0 \mathrm{wt} \% \mathrm{CeNC} /$ ethyl acetate where crystallization and macroscopic phase separation occurred.

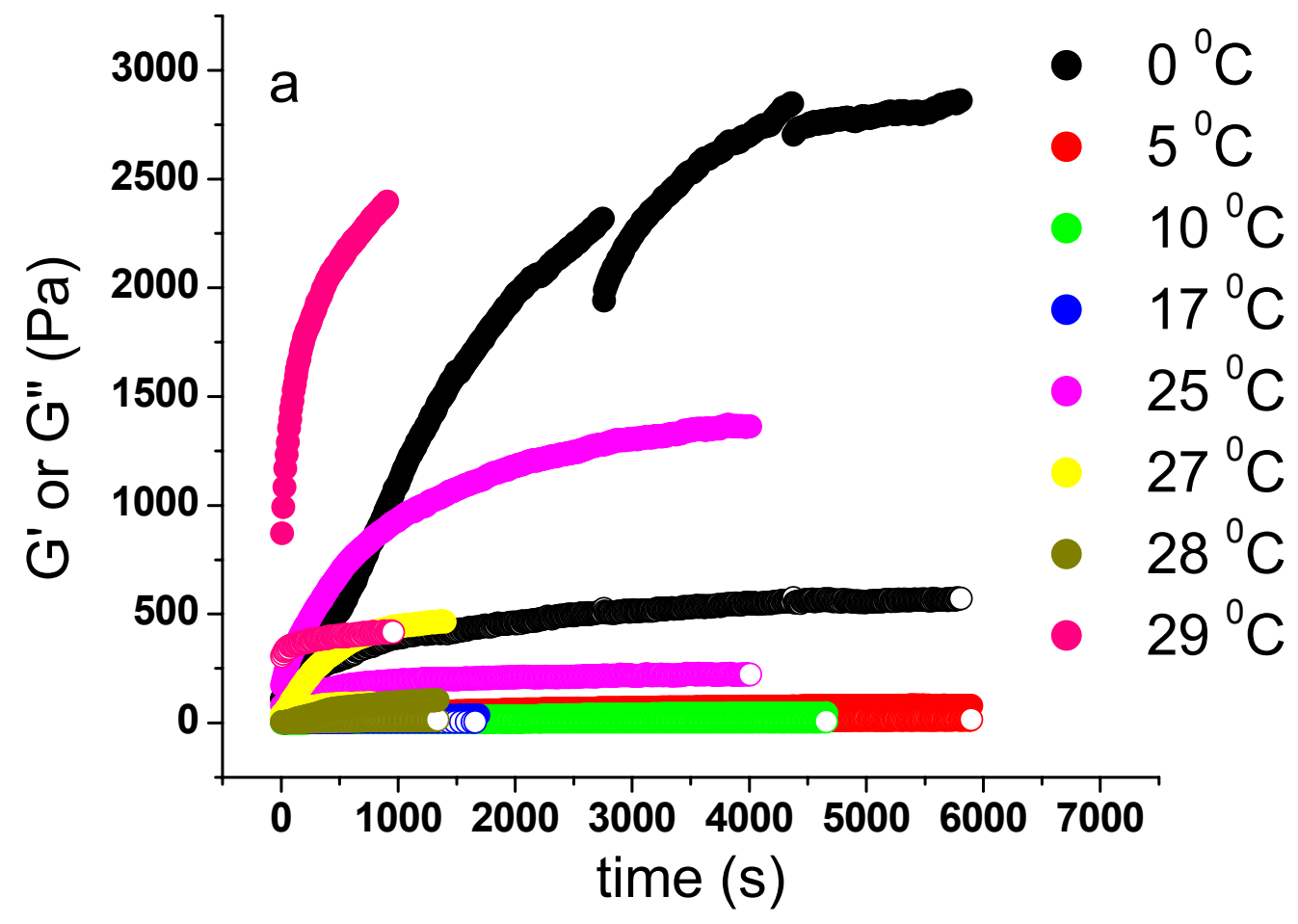



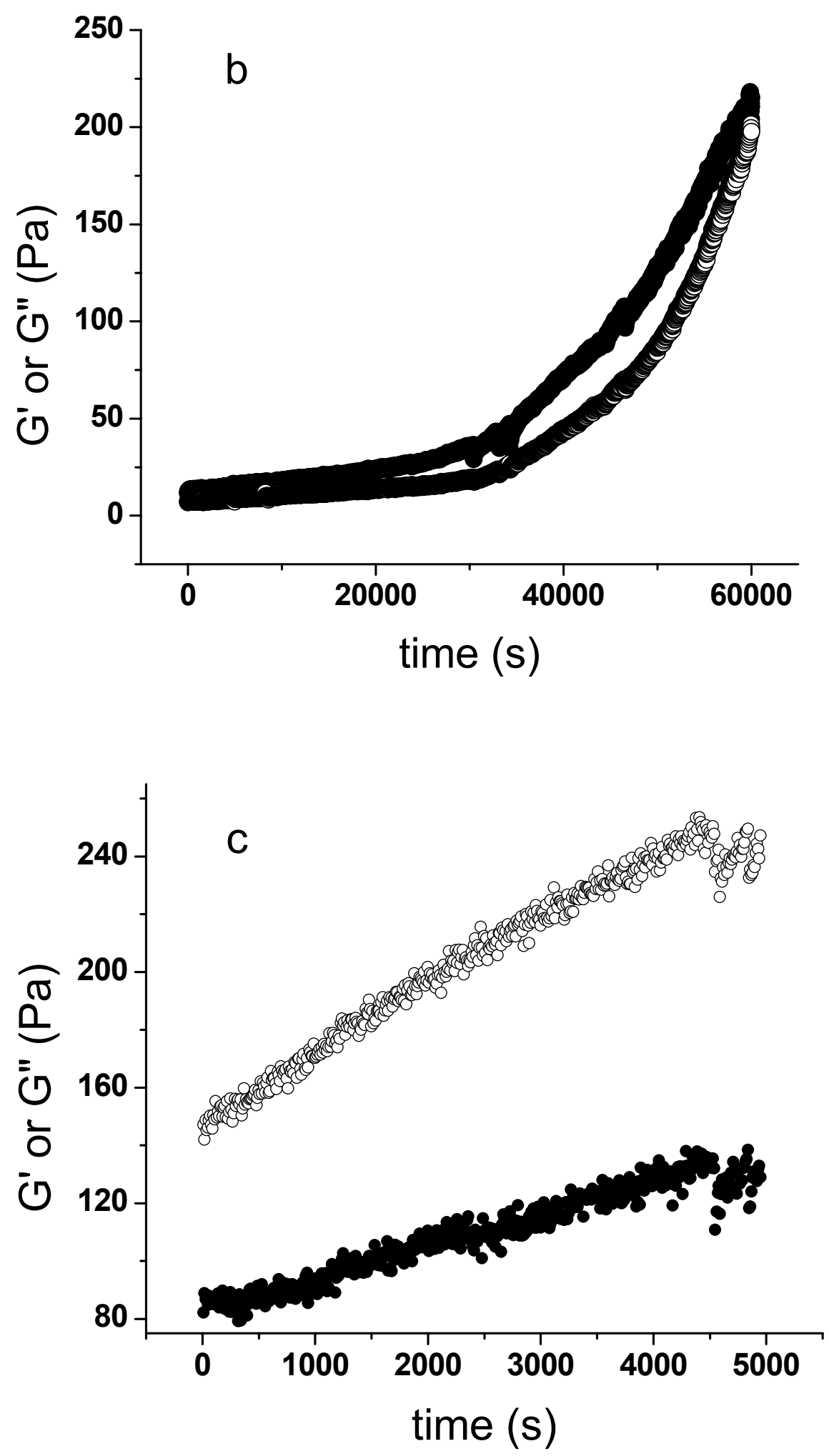

Supporting Figure 8. Recovery of viscoelastic properties of $1.0 \mathrm{wt} \% \mathrm{CNC} / n$-dodecane 
gels (formed by incubation of the sols at 30 and $40{ }^{\circ} \mathrm{C}$, respectively) after being sheared at $2 \mathrm{rad} / \mathrm{s}$ for $600 \mathrm{~s}$ and left at the temperatures shown in a). In b) $\left(30^{\circ} \mathrm{C}\right)$ and c) $\left(40^{\circ} \mathrm{C}\right)$, $G$ ' is filled symbols and $G$ " is open symbols.

Supporting Table 6. Summary of $\tau$ values from single exponential fits of individual $G$ ' and $G$ " values of $1.0 \mathrm{wt} \% \mathbf{C N C} / n$-dodecane gels formed by incubation of the sols at the temperatures indicated and then sheared at $2 \mathrm{rad} / \mathrm{s}$ for $600 \mathrm{~s}$ at the same temperatures.

\begin{tabular}{|l|l|l|}
\hline Temperature $\left( \pm 0.1^{\circ} \mathrm{C}\right)$ & from $G^{\prime}\left(\times 10^{2} \mathrm{~s}\right)$ & from $G^{\prime \prime}\left(\times 10^{2} \mathrm{~s}\right)$ \\
\hline 0.0 & $15.3 \pm 0.2$ & $14.7 \pm 0.2$ \\
\hline 5.0 & $16.0 \pm 0.1$ & $17.9 \pm 0.2$ \\
\hline 10.0 & $29.0 \pm 0.2$ & $29.0 \pm 0.3$ \\
\hline 10.0 & $13.5 \pm 0.3$ & $13.4 \pm 0.3$ \\
\hline 17.0 & $8.39 \pm 0.24$ & $6.03 \pm 0.29$ \\
\hline 25.0 & $8.67 \pm 0.17$ & $6.68 \pm 0.46$ \\
\hline 25.0 & $11.1 \pm 0.2$ & $5.81 \pm 0.18$ \\
\hline 27.0 & $4.22 \pm 0.03$ & $1.94 \pm 0.05$ \\
\hline 27.0 & $4.76 \pm 0.12$ & $1.84 \pm 0.08$ \\
\hline 27.0 & $5.34 \pm 0.13$ & $1.85 \pm 0.04$ \\
\hline 28.0 & $4.29 \pm 0.05$ & $4.12 \pm 0.06$ \\
\hline 28.0 & $3.89 \pm 0.07$ & $4.02 \pm 0.11$ \\
\hline 29.0 & $3.05 \pm 0.04$ & $3.15 \pm 0.07$ \\
\hline
\end{tabular}




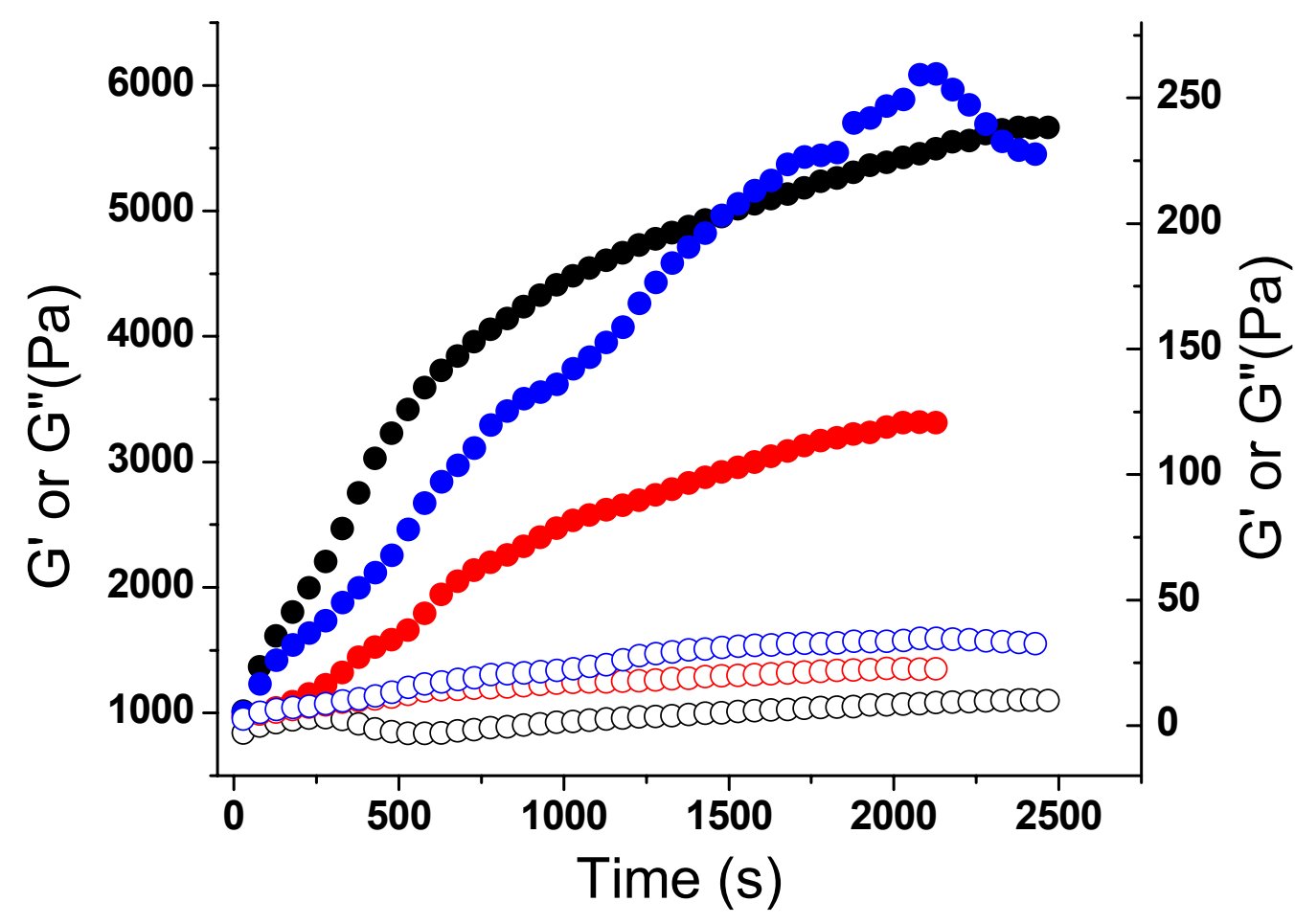

Supporting Figure 9. Recovery of $G^{\prime}$ (filled symbols) and $G$ " (open symbols) of 3.0 $\mathrm{wt} \% \mathrm{CNC} / n$-dodecane gels (formed by incubation of the sols at 0,15 or $30^{\circ} \mathrm{C}$ ) after being sheared at $2 \mathrm{rad} / \mathrm{s}$ for $600 \mathrm{~s}$ at $0\left(\circ, \bullet\right.$; left axis), $15\left(\circ, \bullet\right.$; right axis) and $30^{\circ} \mathrm{C}(\circ$, $\bullet$; right axis). 
Supporting Table 7. SANS data for kinetic runs. Summed counts during $10 \mathrm{~s}$ intervals with $35 \mathrm{~s}$ dead time between intervals.

\begin{tabular}{|c|c|c|c|}
\hline File names & Labels & Date and time & Tot. cnts \\
\hline OCT03207.SA2_RW_E916 & $5 \mathrm{M}$ Scatt KIN2 CNC 3\% 40c & 10/17/2003 9:30 & 18 \\
\hline ОСT03208.SA2_RW_E917 & $5 \mathrm{M}$ Scatt KIN2 CNC 3\% 40c & 10/17/2003 9:40 & 1142 \\
\hline OCT03209.SA2_RW_E918 & 5M Scatt KIN2 CNC 3\% 40c & 10/17/2003 9:41 & 1067 \\
\hline OCT03210.SA2_RW_E919 & 5M Scatt KIN2 CNC 3\% 40c & 10/17/2003 9:42 & 3594 \\
\hline OCT03211.SA2_RW_E920 & $5 \mathrm{M}$ Scatt KIN2 CNC 3\% 40c & 10/17/2003 9:43 & 54922 \\
\hline ОСT03212.SA2_RW_E921 & $5 \mathrm{M}$ Scatt KIN2 CNC 3\% 40c & 10/17/2003 9:44 & 81967 \\
\hline ОСT03213.SA2_RW_E922 & $5 \mathrm{M}$ Scatt KIN2 CNC 3\% 40c & 10/17/2003 9:44 & 87096 \\
\hline OCT03214.SA2_RW_E923 & 5M Scatt KIN2 CNC 3\% 40c & 10/17/2003 9:45 & 89598 \\
\hline OCT03215.SA2_RW_E924 & $5 \mathrm{M}$ Scatt KIN2 CNC 3\% 40c & 10/17/2003 9:46 & 90928 \\
\hline OCT03216.SA2_RW_E925 & $5 \mathrm{M}$ Scatt KIN2 CNC 3\% 40c & 10/17/2003 9:47 & 92230 \\
\hline ОСT03217.SA2_RW_E926 & $5 \mathrm{M}$ Scatt KIN2 CNC 3\% 40c & 10/17/2003 9:47 & 92830 \\
\hline OCT03218.SA2_RW_E927 & 5M Scatt KIN2 CNC 3\% 40c & 10/17/2003 9:48 & 93297 \\
\hline OCT03219.SA2_RW_E928 & $5 \mathrm{M}$ Scatt KIN2 CNC 3\% 40c & 10/17/2003 9:49 & 93350 \\
\hline OCT03220.SA2_RW_E929 & $5 \mathrm{M}$ Scatt KIN2 CNC 3\% 40c & 10/17/2003 9:50 & 93905 \\
\hline OCT03221.SA2_RW_E930 & $5 \mathrm{M}$ Scatt KIN2 CNC 3\% 40c & 10/17/2003 9:51 & 94184 \\
\hline OCT03222.SA2_RW_E931 & 5M Scatt KIN2 CNC 3\% 40c & 10/17/2003 9:51 & 93876 \\
\hline OCT03223.SA2_RW_E932 & $5 \mathrm{M}$ Scatt KIN2 CNC 3\% 40c & 10/17/2003 9:52 & 94709 \\
\hline OCT03224.SA2_RW_E933 & $5 \mathrm{M}$ Scatt KIN2 CNC 3\% 40c & 10/17/2003 9:53 & 94879 \\
\hline OCT03225.SA2_RW_E934 & 5M Scatt KIN2 CNC 3\% 40c & 10/17/2003 9:54 & 93817 \\
\hline OCT03226.SA2_RW_E935 & $5 \mathrm{M}$ Scatt KIN2 CNC 3\% 40c & 10/17/2003 9:54 & 94059 \\
\hline OCT03227.SA2_RW_E936 & 5M Scatt KIN2 CNC 3\% 40c & 10/17/2003 9:55 & 94643 \\
\hline OCT03228.SA2_RW_E937 & 5M Scatt KIN2 CNC 3\% 40c & 10/17/2003 9:56 & 94552 \\
\hline OCT03229.SA2_RW_E938 & $5 \mathrm{M}$ Scatt KIN2 CNC 3\% 40c & 10/17/2003 9:57 & 95486 \\
\hline OCT03230.SA2_RW_E939 & $5 \mathrm{M}$ Scatt KIN2 CNC 3\% 40c & 10/17/2003 9:58 & 94875 \\
\hline ОСT03231.SA2_RW_E940 & $5 \mathrm{M}$ Scatt KIN2 CNC 3\% 40c & 10/17/2003 9:58 & 94638 \\
\hline OCT03232.SA2_RW_E941 & 5M Scatt KIN2 CNC 3\% 40c & 10/17/2003 9:59 & 94142 \\
\hline OCT03233.SA2_RW_E942 & $5 \mathrm{M}$ Scatt KIN2 CNC 3\% 40c & 10/17/2003 10:00 & 94764 \\
\hline OCT03234.SA2_RW_E943 & 5M Scatt KIN2 CNC 3\% 40c & 10/17/2003 10:08 & 94645 \\
\hline ОСT03235.SA2_RW_E944 & $5 \mathrm{M}$ Scatt KIN2 CNC 3\% 40c & $10 / 17 / 2003$ 10:09 & 94597 \\
\hline OCT03236.SA2_RW_E945 & 5M Scatt KIN2 CNC 3\% 40c & 10/17/2003 10:10 & 95577 \\
\hline OCT03237.SA2_RW_E946 & 5M Scatt KIN2 CNC 3\% 40c & 10/17/2003 10:11 & 95381 \\
\hline OCT03238.SA2_RW_E947 & $5 \mathrm{M}$ Scatt KIN2 CNC 3\% 40c & 10/17/2003 10:11 & 94927 \\
\hline OCT03239.SA2_RW_E948 & 5M Scatt KIN2 CNC 3\% 40c & 10/17/2003 10:12 & 95178 \\
\hline ОСT03240.SA2_RW_E949 & $5 \mathrm{M}$ Scatt KIN2 CNC 3\% 40c & 10/17/2003 10:13 & 95102 \\
\hline OCT03241.SA2_RW_E950 & 5M Scatt KIN2 CNC 3\% 40c & 10/17/2003 10:14 & 95296 \\
\hline OCT03242.SA2_RW_E951 & $5 \mathrm{M}$ Scatt KIN2 CNC 3\% 40c & 10/17/2003 10:14 & 94915 \\
\hline OCT03243.SA2_RW_E952 & $5 \mathrm{M}$ Scatt KIN2 CNC 3\% 40c & 10/17/2003 10:15 & 94590 \\
\hline
\end{tabular}


OCT03244.SA2_RW_E953 OCT03245.SA2_RW_E954 OCT03246.SA2_RW_E955 OCT03247.SA2_RW_E956 OCT03248.SA2_RW_E957 OCT03249.SA2_RW_E958 OCT03250.SA2_RW_E959 OCT03251.SA2_RW_E960 OCT03252.SA2_RW_E961 OCT03253.SA2_RW_E962 OCT03254.SA2_RW_E963 OCT03255.SA2_RW_E964 OCT03256.SA2_RW_E965 OCT03257.SA2_RW_E966 OCT03258.SA2_RW_E967 OCT03259.SA2_RW_E968 OCT03260.SA2_RW_E969 OCT03261.SA2_RW_E970 OCT03262.SA2_RW_E971 OCT03263.SA2_RW_E972 OCT03264.SA2_RW_E973 OCT03265.SA2_RW_E974 OCT03266.SA2_RW_E975 OCT03267.SA2_RW_E976 OCT03268.SA2_RW_E977 OCT03269.SA2_RW_E978 OCT03270.SA2_RW_E979 OCT03271.SA2_RW_E980 OCT03272.SA2_RW_E981 OCT03273.SA2_RW_E982 OCT03274.SA2_RW_E983 OCT03275.SA2_RW_E984 OCT03276.SA2_RW_E985 OCT03277.SA2_RW_E986 OCT03278.SA2_RW_E987 OCT03279.SA2_RW_E988 OCT03280.SA2_RW_E989 OCT03281.SA2_RW_E990 OCT03282.SA2_RW_E991 OCT03283.SA2_RW_E992 OCT03284.SA2_RW_E993
5M Scatt KIN2 CNC 3\% 40c 5M Scatt KIN2 CNC 3\% 40c $5 \mathrm{M}$ Scatt KIN2 CNC 3\% 40c $5 \mathrm{M}$ Scatt KIN2 CNC 3\% 40c $5 \mathrm{M}$ Scatt KIN2 CNC 3\% 40c $5 \mathrm{M}$ Scatt KIN2 CNC 3\% 40c $5 \mathrm{M}$ Scatt KIN2 CNC 3\% 40c $5 \mathrm{M}$ Scatt KIN2 CNC 3\% 40c $5 \mathrm{M}$ Scatt KIN2 CNC 3\% 40c $5 \mathrm{M}$ Scatt KIN2 CNC 3\% 40c $5 \mathrm{M}$ Scatt KIN2 CNC 3\% 40c $5 \mathrm{M}$ Scatt KIN2 CNC 3\% 40c $5 \mathrm{M}$ Scatt KIN2 CNC 3\% 40c $5 \mathrm{M}$ Scatt KIN2 CNC 3\% 40c $5 \mathrm{M}$ Scatt KIN2 CNC 3\% 40c $5 \mathrm{M}$ Scatt KIN2 CNC 3\% 40c $5 \mathrm{M}$ Scatt KIN2 CNC 3\% 40c $5 \mathrm{M}$ Scatt KIN2 CNC 3\% 40c $5 \mathrm{M}$ Scatt KIN2 CNC 3\% 40c $5 \mathrm{M}$ Scatt KIN2 CNC 3\% 40c $5 \mathrm{M}$ Scatt KIN2 CNC 3\% 40c 5M Scatt KIN2 CNC 3\% 40c $5 \mathrm{M}$ Scatt KIN2 CNC 3\% 40c $5 \mathrm{M}$ Scatt KIN2 CNC 3\% 40c $5 \mathrm{M}$ Scatt KIN2 CNC 3\% 40c $5 \mathrm{M}$ Scatt KIN2 CNC 3\% 40c $5 \mathrm{M}$ Scatt KIN2 CNC 3\% 40c $5 \mathrm{M}$ Scatt KIN2 CNC 3\% 40c $5 \mathrm{M}$ Scatt KIN2 CNC 3\% 40c $5 \mathrm{M}$ Scatt KIN2 CNC 3\% 40c $5 \mathrm{M}$ Scatt KIN2 CNC 3\% 40c $5 \mathrm{M}$ Scatt KIN2 CNC 3\% 40c $5 \mathrm{M}$ Scatt KIN2 CNC 3\% 40c $5 \mathrm{M}$ Scatt KIN2 CNC 3\% 40c $5 \mathrm{M}$ Scatt KIN2 CNC 3\% 40c $5 \mathrm{M}$ Scatt KIN2 CNC 3\% 40c $5 \mathrm{M}$ Scatt KIN2 CNC 3\% 40c $5 \mathrm{M}$ Scatt KIN2 CNC 3\% 40c $5 \mathrm{M}$ Scatt KIN2 CNC 3\% 40c 5M Scatt KIN2 CNC 3\% 40c $5 \mathrm{M}$ Scatt KIN2 CNC 3\% 40c
10/17/2003 10:16 95307 10/17/2003 10:29 95355 $10 / 17 / 200310: 30 \quad 95374$ 10/17/2003 10:30 95383 10/17/2003 10:31 95419 10/17/2003 10:32 95381 10/17/2003 10:33 95618 10/17/2003 10:47 95742 10/17/2003 10:47 96035 10/17/2003 10:48 95971 10/17/2003 10:49 96575 10/17/2003 10:50 95293 10/17/2003 10:50 95791 10/17/2003 10:59 1091 10/17/2003 11:00 1059 10/17/2003 11:01 1138 10/17/2003 11:01 7163 10/17/2003 11:02 65098 $10 / 17 / 2003$ 11:03 81951 10/17/2003 11:04 85692 $10 / 17 / 200311: 04 \quad 89038$ $10 / 17 / 200311: 05 \quad 90151$ 10/17/2003 11:06 91426 10/17/2003 11:07 92312 10/17/2003 11:08 92837 10/17/2003 11:08 93219 10/17/2003 11:09 93049 10/17/2003 11:10 93263 10/17/2003 11:11 93387 10/17/2003 11:11 93566 10/17/2003 11:12 94525 10/17/2003 11:13 94292 10/17/2003 11:14 94593 10/17/2003 11:14 94291 10/17/2003 11:15 94602 10/17/2003 11:16 94953 10/17/2003 11:17 94475 10/17/2003 11:18 94224 10/17/2003 11:18 95089 10/17/2003 11:30 95052 10/17/2003 11:30 95641 
OCT03285.SA2_RW_E994 OCT03286.SA2_RW_E995 OCT03287.SA2_RW_E996 OCT03288.SA2_RW_E997 OCT03289.SA2_RW_E998 OCT03290.SA2_RW_E999 OCT03291.SA2_RW_F000 OCT03292.SA2_RW_F001 OCT03293.SA2_RW_F002 OCT03294.SA2_RW_F003 OCT03295.SA2_RW_F004 OCT03296.SA2_RW_F005 OCT03297.SA2_RW_F006 OCT03298.SA2_RW_F007 OCT03299.SA2_RW_F008 OCT03300.SA2_RW_F009 OCT03301.SA2_RW_F010 OCT03302.SA2_RW_F011 OCT03303.SA2_RW_F012 OCT03304.SA2_RW_F013 OCT03305.SA2_RW_F014 OCT03306.SA2_RW_F015 OCT03307.SA2_RW_F016 OCT03308.SA2_RW_F017 OCT03309.SA2_RW_F018 OCT03310.SA2_RW_F019 OCT03311.SA2_RW_F020 OCT03312.SA2_RW_F021 OCT03313.SA2_RW_F022 OCT03314.SA2_RW_F023 OCT03315.SA2_RW_F024 OCT03316.SA2_RW_F025 OCT03317.SA2_RW_F026 OCT03318.SA2_RW_F027 OCT03319.SA2_RW_F028 OCT03320.SA2_RW_F029 OCT03321.SA2_RW_F030 OCT03322.SA2_RW_F031 OCT03323.SA2_RW_F032 OCT03324.SA2_RW_F033 OCT03325.SA2_RW_F034
5M Scatt KIN2 CNC 3\% 40c $5 \mathrm{M}$ Scatt KIN2 CNC 3\% 40c $5 \mathrm{M}$ Scatt KIN2 CNC 3\% 40c 5M Scatt KIN2 CNC 3\% 40c $5 \mathrm{M}$ Scatt KIN2 CNC 3\% 40c $5 \mathrm{M}$ Scatt KIN2 CNC 3\% 40c $5 \mathrm{M}$ Scatt KIN2 CNC 3\% 40c 5M Scatt KIN2 CNC 3\% 40c 5M Scatt KIN2 CNC 3\% 40c 5M Scatt KIN2 CNC 3\% 40c 5M Scatt KIN2 CNC 3\% 40c $5 \mathrm{M}$ Scatt KIN2 CNC 3\% 40c 5M Scatt KIN2 CNC 3\% 40c $5 \mathrm{M}$ Scatt KIN2 CNC 3\% 40c 5M Scatt KIN2 CNC 3\% 40c 5M Scatt KIN2 CNC 3\% 40c $5 \mathrm{M}$ Scatt KIN2 CNC 3\% 40c 5M Scatt KIN2 CNC 3\% 40c 5M Scatt KIN2 CNC 3\% 40c $5 \mathrm{M}$ Scatt KIN2 CNC 3\% 40c 5M Scatt KIN2 CNC 3\% 40c 5M Scatt KIN2 CNC 3\% 40c 5M Scatt KIN2 CNC 3\% 40c $5 \mathrm{M}$ Scatt KIN2 CNC 3\% 40c 5M Scatt KIN2 CNC 3\% 40c $5 \mathrm{M}$ Scatt KIN2 CNC 3\% 40c $5 \mathrm{M}$ Scatt KIN2 CNC 3\% 40c $5 \mathrm{M}$ Scatt KIN2 CNC 3\% 40c 5M Scatt KIN2 CNC 3\% 40c 5M Scatt KIN2 CNC 3\% 40c $5 \mathrm{M}$ Scatt KIN2 CNC 3\% 40c $5 \mathrm{M}$ Scatt KIN2 CNC 3\% 40c $5 \mathrm{M}$ Scatt KIN2 CNC 3\% 40c $5 \mathrm{M}$ Scatt KIN2 CNC 3\% 40c $5 \mathrm{M}$ Scatt KIN2 CNC 3\% 40c 5M Scatt KIN2 CNC 3\% 40c $5 \mathrm{M}$ Scatt KIN2 CNC 3\% 40c $5 \mathrm{M}$ Scatt KIN CNC 1\% 40c $5 \mathrm{M}$ Scatt KIN CNC 1\% 40c $5 \mathrm{M}$ Scatt KIN CNC 1\% 40c $5 \mathrm{M}$ Scatt KIN CNC 1\% 40c
10/17/2003 11:31 10/17/2003 11:32 $10 / 17 / 200311: 33$ 10/17/2003 11:33 10/17/2003 11:34 $10 / 17 / 200311: 35$ 10/17/2003 11:36 10/17/2003 11:36 10/17/2003 11:37 10/17/2003 12:10 10/17/2003 12:11 10/17/2003 12:11 10/17/2003 12:12 10/17/2003 12:13 10/17/2003 12:14 $10 / 17 / 200312: 14$ 10/17/2003 12:15 10/17/2003 12:16 10/17/2003 12:17 10/17/2003 12:17 $10 / 17 / 2003$ 12:28 $10 / 17 / 200312: 28$ $10 / 17 / 2003$ 12:29 $10 / 17 / 200312: 30$ 10/17/2003 12:31 $10 / 17 / 200312: 31$ 10/17/2003 12:32 $10 / 17 / 200312: 33$ 10/17/2003 12:34 $10 / 17 / 2003$ 12:34 10/17/2003 12:35 $10 / 17 / 200312: 42$ $10 / 17 / 2003$ 12:43 $10 / 17 / 200312: 44$ 10/17/2003 12:45 $10 / 17 / 200312: 46$ $10 / 17 / 2003$ 12:46 $10 / 17 / 200312: 55$ $10 / 17 / 2003$ 12:55 10/17/2003 12:56 10/17/2003 12:57
95458

95800

96311

95669

95505

95654

96321

96215

96221

96760

97056

96447

96861

97412

97315

97273

96928

95793

96279

97164

97245 96820 97463 97556 97627 97709 96580 96952 97308 97114 97538 97689 97592 97497 97200 97189 98019 856 899 893 867 
OCT03326.SA2_RW_F035 OCT03327.SA2_RW_F036 OCT03328.SA2_RW_F037 OCT03329.SA2_RW_F038 OCT03330.SA2_RW_F039 OCT03331.SA2_RW_F040 OCT03332.SA2_RW_F041 OCT03333.SA2_RW_F042 OCT03334.SA2_RW_F043 OCT03335.SA2_RW_F044 OCT03336.SA2_RW_F045 OCT03337.SA2_RW_F046 OCT03338.SA2_RW_F047 OCT03339.SA2_RW_F048 OCT03340.SA2_RW_F049 OCT03341.SA2_RW_F050 OCT03342.SA2_RW_F051 OCT03343.SA2_RW_F052 OCT03344.SA2_RW_F053 OCT03345.SA2_RW_F054 OCT03346.SA2_RW_F055 OCT03347.SA2_RW_F056 OCT03348.SA2_RW_F057 OCT03349.SA2_RW_F058 OCT03350.SA2_RW_F059 OCT03351.SA2_RW_F060 OCT03352.SA2_RW_F061 OCT03353.SA2_RW_F062 OCT03354.SA2_RW_F063 OCT03355.SA2_RW_F064 OCT03356.SA2_RW_F065 OCT03357.SA2_RW_F066 OCT03358.SA2_RW_F067 OCT03359.SA2_RW_F068 OCT03360.SA2_RW_F069 OCT03361.SA2_RW_F070 OCT03362.SA2_RW_F071 OCT03363.SA2_RW_F072 OCT03364.SA2_RW_F073 OCT03365.SA2_RW_F074 OCT03366.SA2_RW_F075
5M Scatt KIN CNC 1\% 40c $5 \mathrm{M}$ Scatt KIN CNC 1\% 40c 5M Scatt KIN CNC 1\% 40c $5 \mathrm{M}$ Scatt KIN CNC 1\% 40c $5 \mathrm{M}$ Scatt KIN CNC 1\% 40c $5 \mathrm{M}$ Scatt KIN CNC 1\% 40c $5 \mathrm{M}$ Scatt KIN CNC 1\% 40c $5 \mathrm{M}$ Scatt KIN CNC 1\% 40c 5M Scatt KIN CNC 1\% 40c $5 \mathrm{M}$ Scatt KIN CNC 1\% 40c $5 \mathrm{M}$ Scatt KIN CNC 1\% 40c 5M Scatt KIN CNC 1\% 40c 5M Scatt KIN CNC 1\% 40c $5 \mathrm{M}$ Scatt KIN CNC 1\% 40c 5M Scatt KIN CNC 1\% 40c $5 \mathrm{M}$ Scatt KIN CNC 1\% 40c $5 \mathrm{M}$ Scatt KIN CNC 1\% 40c $5 \mathrm{M}$ Scatt KIN CNC 1\% 40c 5M Scatt KIN CNC 1\% 40c $5 \mathrm{M}$ Scatt KIN CNC 1\% 40c 5M Scatt KIN CNC 1\% 40c $5 \mathrm{M}$ Scatt KIN CNC 1\% 40c 5M Scatt KIN CNC 1\% 40c $5 \mathrm{M}$ Scatt KIN CNC 1\% 40c 5M Scatt KIN CNC 1\% 40c $5 \mathrm{M}$ Scatt KIN CNC 1\% 40c 5M Scatt KIN CNC 1\% 40c 5M Scatt KIN CNC 1\% 40c 5M Scatt KIN CNC 1\% 40c $5 \mathrm{M}$ Scatt KIN CNC 1\% 40c $5 \mathrm{M}$ Scatt KIN CNC 1\% 40c 5M Scatt KIN CNC 1\% 40c $5 \mathrm{M}$ Scatt KIN CNC 1\% 40c 5M Scatt KIN CNC 1\% 40c $5 \mathrm{M}$ Scatt KIN CNC 1\% 40c 5M Scatt KIN CNC 1\% 40c 5M Scatt KIN CNC 1\% 40c 5M Scatt KIN CNC 1\% 40c 5M Scatt KIN CNC 1\% 40c 5M Scatt KIN CNC 1\% 40c $5 \mathrm{M}$ Scatt KIN CNC 1\% 40c
10/17/2003 12:58 944 $10 / 17 / 200312: 59 \quad 897$ $10 / 17 / 200312: 59 \quad 883$ $10 / 17 / 2003$ 13:00 882 10/17/2003 13:01 874 10/17/2003 13:02 920 10/17/2003 13:02 941 10/17/2003 13:08 1290 10/17/2003 13:09 1306 10/17/2003 13:10 1340 10/17/2003 13:11 1370 10/17/2003 13:11 1471 10/17/2003 13:12 1578 10/17/2003 13:21 2145 10/17/2003 13:21 2227 10/17/2003 13:22 2399 $10 / 17 / 200313: 23 \quad 2375$ 10/17/2003 13:24 2446 10/17/2003 13:24 2569 10/17/2003 13:25 2655 10/17/2003 13:26 2702 10/17/2003 13:27 2767 10/17/2003 13:27 2904 $10 / 17 / 2003$ 13:28 2909 10/17/2003 13:33 3496 10/17/2003 13:34 3530 10/17/2003 13:35 3549 10/17/2003 13:35 3539 10/17/2003 13:36 3682 10/17/2003 13:37 3769 10/17/2003 13:38 3884 10/17/2003 13:38 4054 10/17/2003 13:39 4021 10/17/2003 13:40 4089 10/17/2003 13:41 4230 10/17/2003 13:42 4165 10/17/2003 13:42 4346 10/17/2003 13:43 4399 $10 / 17 / 200313: 44 \quad 4452$ $10 / 17 / 2003$ 13:45 4603 10/17/2003 13:45 4693 
OCT03367.SA2_RW_F076 OCT03368.SA2_RW_F077 OCT03369.SA2_RW_F078 OCT03370.SA2_RW_F079 OCT03371.SA2_RW_F080 OCT03372.SA2_RW_F081 OCT03373.SA2_RW_F082 OCT03374.SA2_RW_F083 OCT03375.SA2_RW_F084 OCT03376.SA2_RW_F085 OCT03377.SA2_RW_F086 OCT03378.SA2_RW_F087 OCT03379.SA2_RW_F088 OCT03380.SA2_RW_F089 OCT03381.SA2_RW_F090 OCT03382.SA2_RW_F091 OCT03383.SA2_RW_F092 OCT03384.SA2_RW_F093 OCT03385.SA2_RW_F094 OCT03386.SA2_RW_F095 OCT03387.SA2_RW_F096 OCT03388.SA2_RW_F097 OCT03389.SA2_RW_F098 OCT03390.SA2_RW_F099 OCT03391.SA2_RW_F100 OCT03392.SA2_RW_F101 OCT03393.SA2_RW_F102 OCT03394.SA2_RW_F103 OCT03395.SA2_RW_F104 OCT03396.SA2_RW_F105 OCT03397.SA2_RW_F106 OCT03398.SA2_RW_F107 OCT03399.SA2_RW_F108 OCT03400.SA2_RW_F109 OCT03401.SA2_RW_F110 OCT03402.SA2_RW_F111 OCT03403.SA2_RW_F112 OCT03404.SA2_RW_F113 OCT03405.SA2_RW_F114 OCT03406.SA2_RW_F115 OCT03407.SA2_RW_F116
5M Scatt KIN CNC 1\% 40c $5 \mathrm{M}$ Scatt KIN CNC 1\% 40c $5 \mathrm{M}$ Scatt KIN CNC 1\% 40c $5 \mathrm{M}$ Scatt KIN CNC 1\% 40c $5 \mathrm{M}$ Scatt KIN CNC 1\% 40c $5 \mathrm{M}$ Scatt KIN CNC 1\% 40c $5 \mathrm{M}$ Scatt KIN CNC 1\% 40c $5 \mathrm{M}$ Scatt KIN CNC 1\% 40c $5 \mathrm{M}$ Scatt KIN CNC 1\% 40c $5 \mathrm{M}$ Scatt KIN CNC 1\% 40c $5 \mathrm{M}$ Scatt KIN CNC 1\% 40c $5 \mathrm{M}$ Scatt KIN CNC 1\% 40c $5 \mathrm{M}$ Scatt KIN CNC 1\% 40c $5 \mathrm{M}$ Scatt KIN CNC 1\% 40c $5 \mathrm{M}$ Scatt KIN CNC 1\% 40c $5 \mathrm{M}$ Scatt KIN CNC 1\% 40c $5 \mathrm{M}$ Scatt KIN CNC 1\% 40c $5 \mathrm{M}$ Scatt KIN CNC 1\% 40c $5 \mathrm{M}$ Scatt KIN CNC 1\% 40c $5 \mathrm{M}$ Scatt KIN CNC 1\% 40c $5 \mathrm{M}$ Scatt KIN CNC 1\% 40c $5 \mathrm{M}$ Scatt KIN CNC 1\% 40c $5 \mathrm{M}$ Scatt KIN CNC 1\% 40c $5 \mathrm{M}$ Scatt KIN CNC 1\% 40c $5 \mathrm{M}$ Scatt KIN CNC 1\% 40c $5 \mathrm{M}$ Scatt KIN CNC 1\% 40c $5 \mathrm{M}$ Scatt KIN CNC 1\% 40c $5 \mathrm{M}$ Scatt KIN CNC 1\% 40c $5 \mathrm{M}$ Scatt KIN CNC 1\% 40c $5 \mathrm{M}$ Scatt KIN CNC 1\% 40c $5 \mathrm{M}$ Scatt KIN CNC 1\% 40c $5 \mathrm{M}$ Scatt KIN CNC 1\% 40c $5 \mathrm{M}$ Scatt KIN CNC 1\% 40c $5 \mathrm{M}$ Scatt KIN CNC 1\% 40c $5 \mathrm{M}$ Scatt KIN CNC 1\% 40c $5 \mathrm{M}$ Scatt KIN CNC 1\% 40c $5 \mathrm{M}$ Scatt KIN CNC 1\% 40c $5 \mathrm{M}$ Scatt KIN CNC 1\% 40c $5 \mathrm{M}$ Scatt KIN CNC 1\% 40c $5 \mathrm{M}$ Scatt KIN CNC 1\% 40c $5 \mathrm{M}$ Scatt KIN CNC 1\% 40c
10/17/2003 13:46 4788

10/17/2003 13:47 4699

$10 / 17 / 200313: 48 \quad 4869$

$10 / 17 / 200313: 49 \quad 4904$

$10 / 17 / 200313: 56 \quad 5815$

$10 / 17 / 200313: 59 \quad 5947$

$10 / 17 / 200314: 01 \quad 6270$

$10 / 17 / 200314: 04 \quad 6446$

10/17/2003 14:07 6695

10/17/2003 14:09 7019

10/17/2003 14:12 7179

10/17/2003 14:14 7604

10/17/2003 14:17 7739

$10 / 17 / 200314: 19 \quad 8010$

$10 / 17 / 200314: 22 \quad 8400$

$10 / 17 / 200314: 25 \quad 8621$

$10 / 17 / 200314: 28 \quad 8876$

$10 / 17 / 200314: 29 \quad 8852$

$10 / 17 / 200314: 329395$

10/17/2003 14:34 9532

10/17/2003 14:37 9772

10/17/2003 14:39 9722

$10 / 17 / 200314: 42 \quad 10010$

$10 / 17 / 200314: 44 \quad 10355$

$10 / 17 / 200314: 47 \quad 10608$

$10 / 17 / 200315: 05 \quad 12230$

$10 / 17 / 2003$ 15:06 12291

$10 / 17 / 200315: 06 \quad 12257$

10/17/2003 15:07 12472

10/17/2003 15:19 13503

$10 / 17 / 200315: 20 \quad 13616$

$10 / 17 / 200315: 21 \quad 13658$

$10 / 17 / 200315: 22 \quad 13556$

$10 / 17 / 200315: 22 \quad 13808$

$10 / 17 / 200315: 23 \quad 13878$

$10 / 17 / 200315: 24 \quad 13873$

$10 / 17 / 200315: 25 \quad 13884$

$10 / 17 / 200315: 25 \quad 13945$

$10 / 17 / 200315: 42 \quad 15254$

$10 / 17 / 200315: 43 \quad 14964$

$10 / 17 / 200315: 44 \quad 15218$ 
OCT03408.SA2_RW_F117 OCT03409.SA2_RW_F118 OCT03410.SA2_RW_F119 OCT03411.SA2_RW_F120 OCT03412.SA2_RW_F121 OCT03413.SA2_RW_F122 OCT03414.SA2_RW_F123 OCT03415.SA2_RW_F124 OCT03416.SA2_RW_F125 OCT03417.SA2_RW_F126 OCT03418.SA2_RW_F127 OCT03419.SA2_RW_F128 OCT03420.SA2_RW_F129 OCT03421.SA2_RW_F130 OCT03422.SA2_RW_F131 OCT03423.SA2_RW_F132 OCT03424.SA2_RW_F133 OCT03425.SA2_RW_F134 OCT03426.SA2_RW_F135 OCT03427.SA2_RW_F136 OCT03428.SA2_RW_F137 OCT03429.SA2_RW_F138 OCT03430.SA2_RW_F139 OCT03431.SA2_RW_F140 OCT03432.SA2_RW_F141 OCT03433.SA2_RW_F142 OCT03434.SA2_RW_F143 OCT03435.SA2_RW_F144 OCT03436.SA2_RW_F145 OCT03437.SA2_RW_F146 OCT03438.SA2_RW_F147 OCT03439.SA2_RW_F148 OCT03440.SA2_RW_F149 OCT03441.SA2_RW_F150 OCT03442.SA2_RW_F151 OCT03443.SA2_RW_F152 OCT03444.SA2_RW_F153 OCT03445.SA2_RW_F154 OCT03446.SA2_RW_F155 OCT03447.SA2_RW_F156 OCT03448.SA2_RW_F157
5M Scatt KIN CNC 1\% 40c $5 \mathrm{M}$ Scatt KIN CNC 1\% 40c 5M Scatt KIN CNC 1\% 40c $5 \mathrm{M}$ Scatt KIN CNC 1\% 40c $5 \mathrm{M}$ Scatt KIN CNC 1\% 40c $5 \mathrm{M}$ Scatt KIN CNC 1\% 40c $5 \mathrm{M}$ Scatt KIN CNC 1\% 40c $5 \mathrm{M}$ Scatt KIN CNC 1\% 40c 5M Scatt KIN CNC 1\% 40c $5 \mathrm{M}$ Scatt KIN CNC 1\% 40c $5 \mathrm{M}$ Scatt KIN CNC 1\% 40c $5 \mathrm{M}$ Scatt KIN CNC 1\% 40c 5M Scatt KIN CNC 1\% 40c $5 \mathrm{M}$ Scatt KIN CNC 1\% 40c 5M Scatt KIN CNC 1\% 40c $5 \mathrm{M}$ Scatt KIN CNC 1\% 40c $5 \mathrm{M}$ Scatt KIN CNC 1\% 40c $5 \mathrm{M}$ Scatt KIN CNC 1\% 40c 5M Scatt KIN CNC 1\% 40c $5 \mathrm{M}$ Scatt KIN CNC 1\% 40c 5M Scatt KIN CNC 1\% 40c $5 \mathrm{M}$ Scatt KIN CNC 1\% 40c 5M Scatt KIN CNC 1\% 40c $5 \mathrm{M}$ Scatt KIN CNC 1\% 40c 5M Scatt KIN CNC 1\% 40c $5 \mathrm{M}$ Scatt KIN CNC 1\% 40c 5M Scatt KIN CNC 1\% 40c 5M Scatt KIN CNC 1\% 40c 5M Scatt KIN CNC 1\% 40c $5 \mathrm{M}$ Scatt KIN CNC 1\% 40c 5M Scatt KIN CNC 1\% 40c $5 \mathrm{M}$ Scatt KIN CNC 1\% 40c 5M Scatt KIN CNC 1\% 40c 5M Scatt KIN CNC 1\% 40c 5M Scatt KIN CNC 1\% 40c $5 \mathrm{M}$ Scatt KIN CNC 1\% 40c 5M Scatt KIN CNC 1\% 40c 5M Scatt KIN CNC 1\% 40c 5M Scatt KIN CNC 1\% 40c 5M Scatt KIN CNC 1\% 40c $5 \mathrm{M}$ Scatt KIN CNC 1\% 40c
10/17/2003 15:45 15258 10/17/2003 15:45 15367 10/17/2003 15:46 15355 10/17/2003 16:07 16983 10/17/2003 16:08 16844 10/17/2003 16:09 16879 10/17/2003 16:10 17047 10/17/2003 16:22 17489 10/17/2003 16:22 17900 10/17/2003 16:23 17839 10/17/2003 16:24 17953 10/17/2003 16:25 17900 10/17/2003 16:26 18042 10/17/2003 16:45 19086 10/17/2003 16:46 19056 10/17/2003 16:47 18885 10/17/2003 16:47 18952 10/17/2003 16:48 18979 10/17/2003 16:49 18995 10/17/2003 17:02 19983 10/17/2003 17:03 19746 10/17/2003 17:04 19814 10/17/2003 17:05 19873 10/17/2003 17:10 19858 10/17/2003 17:10 20276 10/17/2003 17:11 20196 $10 / 17 / 2003$ 17:12 20164 10/17/2003 17:13 20152 10/17/2003 17:13 19924 10/17/2003 17:35 20972 10/17/2003 17:36 21573 10/17/2003 17:36 21188 $10 / 17 / 2003$ 17:37 21023 10/17/2003 17:38 21171 10/17/2003 17:39 21442 $10 / 17 / 200317: 52 \quad 22020$ 10/17/2003 17:52 21505 10/17/2003 17:53 21777 10/17/2003 17:54 21894 $10 / 17 / 200318: 16 \quad 22363$ 10/17/2003 18:17 22699 
OCT03449.SA2_RW_F158 OCT03450.SA2_RW_F159 OCT03451.SA2_RW_F160 OCT03452.SA2_RW_F161 OCT03453.SA2_RW_F162 OCT03454.SA2_RW_F163 OCT03455.SA2_RW_F164 OCT03456.SA2_RW_F165 OCT03457.SA2_RW_F166 OCT03458.SA2_RW_F167 OCT03459.SA2_RW_F168 OCT03460.SA2_RW_F169 OCT03461.SA2_RW_F170 OCT03462.SA2_RW_F171 OCT03463.SA2_RW_F172 OCT03464.SA2_RW_F173 OCT03465.SA2_RW_F174 OCT03466.SA2_RW_F175 OCT03467.SA2_RW_F176 OCT03468.SA2_RW_F177 OCT03469.SA2_RW_F178 OCT03470.SA2_RW_F179 OCT03471.SA2_RW_F180 OCT03472.SA2_RW_F181 OCT03473.SA2_RW_F182 OCT03474.SA2_RW_F183 OCT03475.SA2_RW_F184 OCT03476.SA2_RW_F185 OCT03477.SA2_RW_F186 OCT03478.SA2_RW_F187 OCT03479.SA2_RW_F188 OCT03480.SA2_RW_F189 OCT03481.SA2_RW_F190 OCT03482.SA2_RW_F191 OCT03483.SA2_RW_F192 OCT03484.SA2_RW_F193 OCT03485.SA2_RW_F194 OCT03486.SA2_RW_F195 OCT03487.SA2_RW_F196
5M Scatt KIN CNC 1\% 40c $5 \mathrm{M}$ Scatt KIN CNC 1\% 40c $5 \mathrm{M}$ Scatt KIN CNC 1\% 40c $5 \mathrm{M}$ Scatt KIN CNC 1\% 40c $5 \mathrm{M}$ Scatt KIN CNC 1\% 40c $5 \mathrm{M}$ Scatt KIN CNC 1\% 40c $5 \mathrm{M}$ Scatt KIN CNC 1\% 40c $5 \mathrm{M}$ Scatt KIN CNC 1\% 40c $5 \mathrm{M}$ Scatt KIN CNC 1\% 40c $5 \mathrm{M}$ Scatt KIN CNC 1\% 40c $5 \mathrm{M}$ Scatt KIN CNC 1\% 40c $5 \mathrm{M}$ Scatt KIN CNC 1\% 40c 5M Scatt KIN CNC 1\% 40c $5 \mathrm{M}$ Scatt KIN CNC 1\% 40c 5M Scatt KIN CNC 1\% 40c $5 \mathrm{M}$ Scatt KIN CNC 1\% 40c $5 \mathrm{M}$ Scatt KIN CNC 1\% 40c $5 \mathrm{M}$ Scatt KIN CNC 1\% 40c 5M Scatt KIN CNC 1\% 40c $5 \mathrm{M}$ Scatt KIN CNC 1\% 40c 5M Scatt KIN CNC 1\% 40c $5 \mathrm{M}$ Scatt KIN CNC 1\% 40c 5M Scatt KIN CNC 1\% 40c $5 \mathrm{M}$ Scatt KIN CNC 1\% 40c $5 \mathrm{M}$ Scatt KIN CNC 1\% 40c $5 \mathrm{M}$ Scatt KIN CNC 1\% 40c 5M Scatt KIN CNC 1\% 40c 5M Scatt KIN CNC 1\% 40c $5 \mathrm{M}$ Scatt KIN CNC 1\% 40c $5 \mathrm{M}$ Scatt KIN CNC 1\% 40c 5M Scatt KIN CNC 1\% 40c $5 \mathrm{M}$ Scatt KIN CNC 1\% 40c 5M Scatt KIN CNC 1\% 40c $5 \mathrm{M}$ Scatt KIN CNC 1\% 40c 5M Scatt KIN CNC 1\% 40c $5 \mathrm{M}$ Scatt KIN CNC 1\% 40c 5M Scatt KIN CNC 1\% 40c $5 \mathrm{M}$ Scatt BANJO CELL EMPTY KINETICS 5M Scatt DODECANE BANJO CELL KINETICS 10/17/2003 19:48 10178

10/17/2003 18:17 22254 10/17/2003 18:18 22874 $10 / 17 / 200318: 1922737$ 10/17/2003 18:20 22571 10/17/2003 18:24 22919 $10 / 17 / 200318: 25 \quad 22713$ 10/17/2003 18:26 22756 10/17/2003 18:27 22995 $10 / 17 / 2003$ 18:27 22954 10/17/2003 18:28 22877 $10 / 17 / 200318: 29 \quad 22785$ $10 / 17 / 200318: 30 \quad 22884$ $10 / 17 / 200318: 30 \quad 22998$ 10/17/2003 18:31 23105 10/17/2003 18:32 23036 10/17/2003 18:48 23090 10/17/2003 18:49 23297 10/17/2003 18:49 23550 $10 / 17 / 200318: 50 \quad 23342$ 10/17/2003 18:51 23367 10/17/2003 18:52 23486 10/17/2003 18:52 23309 10/17/2003 18:53 23429 10/17/2003 18:54 23409 10/17/2003 18:55 23299 10/17/2003 18:55 23478 10/17/2003 19:21 24406 10/17/2003 19:22 24467 10/17/2003 19:22 24279 10/17/2003 19:23 24553 10/17/2003 19:24 24241 10/17/2003 19:25 24277 $10 / 17 / 2003$ 19:25 24463 10/17/2003 19:26 24398 $10 / 17 / 2003$ 19:27 24284 10/17/2003 19:28 24313 10/17/2003 19:29 24421 10/17/2003 20:00 36933 


$\begin{array}{lllll} & \text { 5M Trans } & \text { BANJO CELL } & & \\ \text { OCT03488.SA2_RW_F197 } & \text { CNC DODEC 1\% 25000CTS } & 10 / 17 / 2003 \text { 20:12 } & 102520 \\ & \text { 5M Trans CNC 3\% DODEC } & & \\ \text { OCT03495.SA2_RW_F204 } & \text { SLOWLY COOLED BANJO } & 10 / 17 / 2003 \text { 20:33 } & 99063 \\ & \text { 5M Scatt CNC 3\% DODEC } & & \\ & \text { SLOWLY COOLED BANJO } & & \\ \text { OCT03502.SA2_RW_F211 } & \text { CELL } & & 10 / 17 / 2003 \text { 20:59 } & 7.79 E+06\end{array}$

Supporting Figure 10. Scattering intensity versus $Q$ from data obtained in a $10 \mathrm{~s}$ interval after a 1.0 wt $\%$ CNC in $n$-dodecane- $d_{26}$ sample had remained at $40{ }^{\circ} \mathrm{C}$ for $368 \mathrm{~s}(1), 1824 \mathrm{~s}$ (2), $3855 \mathrm{~s}$ (3), $10058 \mathrm{~s}(4)$, and $33536 \mathrm{~s}$. Note that two oscillations can be detected even with the $10 \mathrm{~s}$ collection time in curve (5).

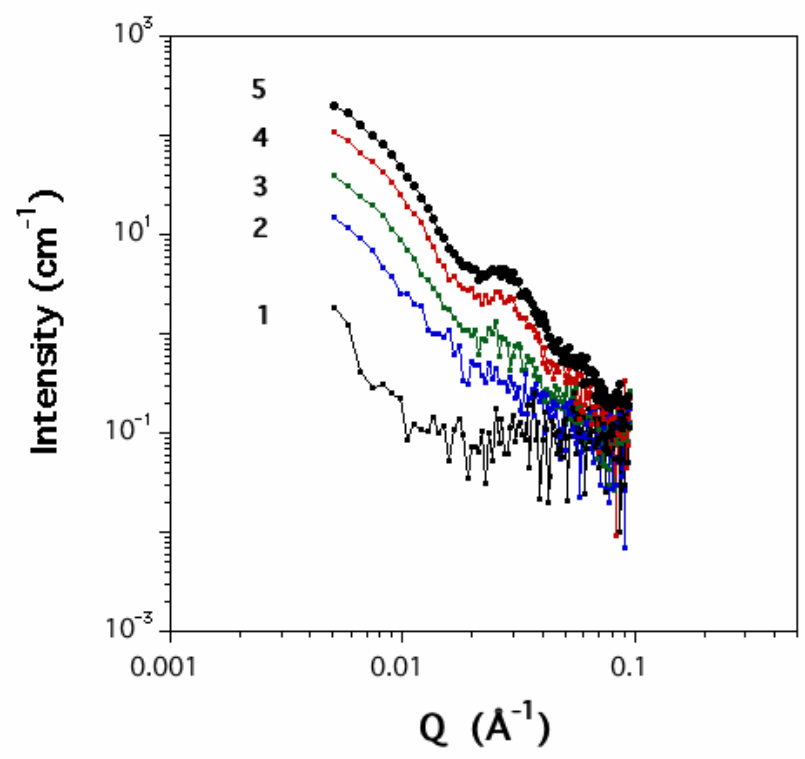

Appendix 1. Derivation of fractal model equation.

Our analysis of the fractal nature of our molecular organogels is based upon a kinetic model developed by Dickinson. ${ }^{7}$ The conditions under which it should apply to $\mathbf{C N C}$ and CeNC organogels are described in the text.

Then, the average size of aggregates $\left(V_{a}\right)$ can be expressed as in eq. 1 , where $V_{n}$ is average volume of nucleated molecules, $V_{0}$ is the volume of a gelator molecule, $N_{0}$ is 
the concentration of gelator ( $\mathrm{mol} /$ volume), $\mathrm{k}$ is rate constant, $f(t)$ is a time function for Smoluchowski-type aggregation, (i.e., $f(t)=t$ where $t$ is time).

$V_{a}=V_{n}+N_{0} \times k \times V_{0} \times f(t)$

When $t$ is sufficiently large, $V_{n}<<N_{0} \times k \times V_{0} \times f(t)$ and eq. 1 becomes eq. 2 .

$V_{a}=N_{0} \times k \times V_{0} \times t$

The effective volume fraction of aggregates at time $t, \phi_{\text {eff }}^{t}$, is proportional to the product of $\rho_{0}$ (the density of nucleated molecules, which is determined by gelation temperature, gelator concentration, etc.) and $R_{a}$ (the average hard-sphere radius of the aggregates) divided by $V_{a}$ (eq. 3).

$\phi_{e f f}^{t} \propto \rho_{0} \times R_{a}^{3} / V_{a}$

In fractal concepts, $R_{a} \propto V_{a}^{1 / D_{f}}$, so that eq. 3 can be rewritten as eq. 4 .

$\phi_{e f f}^{t} \propto \rho_{0} \times V_{a}^{\left(3-D_{f}\right) / D_{f}}$

Substituting eq. 2 into eq. 4, and changing to a logarithmic form yields eq. 5 the equation we have applied in our analyses.

$\ln \phi_{e f f}^{t}=C+\left(3-D_{f}\right) / D_{f} \ln t$

${ }^{1}$ Lu, L.; Cocker, M.; Bachman, R. E.; Weiss, R. G. Langmuir 2000, 16, 20-34.

${ }^{2}$ Lu, L.; Cocker, M.; Bachman, R. E.; Weiss, R. G. Langmuir 2000, 16, 20-34.

${ }^{3}$ Eldridge, J. E.; Ferry, J. D. J. Phys. Chem. 1954, 58, 992-995.

${ }^{4}$ Khan, S. A.; Royer J. R.; Raghavan, S. R. Aviation Fuels with Improved Fire Safety: A Proceeding; National Academy Press: Washington, DC, 1997, pp 31-46.

5 a) Almdal, K.; Dyre, J.; Hvidt, S.; Kramer, O. Polym. Gels Networks 1993, 1, 5-17. b) Khan, S. A.; Royer J. R.; Raghavan, S. R. Aviation Fuels with Improved Fire Safety: A Proceeding; National Academy Press: Washington, DC, 1997, pp 31-46. 
${ }^{6}$ Huang, X.; Terech, P.; Raghavan, S. R.; Weiss, R. G. J. Am. Chem. Soc., 2005, 127, 4336-4344

7 Dickinson, E. J. Chem. Soc., Faraday Trans., 1997, 93, 111-114. 